\title{
Gluconeogenic enzyme PCK1 deficiency promotes CHK2 0 -GIcNAcylation and hepatocellular carcinoma growth upon glucose deprivation
}

\author{
Jin Xiang, ${ }^{1}$ Chang Chen, ${ }^{2}$ Rui Liu, ${ }^{1}$ Dongmei Gou, ${ }^{1}$ Lei Chang, ${ }^{3}$ Haijun Deng, ${ }^{1}$ Qingzhu Gao, ${ }^{1}$ Wanjun Zhang, ${ }^{3}$ Lin Tuo, ${ }^{4}$ \\ Xuanming Pan, ${ }^{1}$ Li Liang, ${ }^{1}$ Jie Xia, ${ }^{1}$ Luyi Huang, ${ }^{1}$ Ke Yao, ${ }^{5}$ Bohong Wang, ${ }^{5}$ Zeping Hu, ${ }^{5}$ Ailong Huang, ${ }^{1}$ Kai Wang, ${ }^{1}$ and Ni Tang ${ }^{1}$ \\ ${ }^{1}$ Key Laboratory of Molecular Biology for Infectious Diseases, Ministry of Education, Institute for Viral Hepatitis, Department of Infectious Diseases, The Second Affiliated Hospital, and ${ }^{2}$ Institute of Life \\ Sciences, Chongqing Medical University, Chongqing, China. ${ }^{3}$ State Key Laboratory of Proteomics, Beijing Proteome Research Center, National Center for Protein Sciences, Beijing Institute of Lifeomics, \\ Beijing, China. ${ }^{4}$ Sichuan Provincial People's Hospital, Sichuan, China. ${ }^{5}$ School of Pharmaceutical Sciences, Tsinghua University, Beijing, China.
}

\begin{abstract}
Although cancer cells are frequently faced with a nutrient- and oxygen-poor microenvironment, elevated hexosaminebiosynthesis pathway (HBP) activity and protein O-GIcNAcylation (a nutrient sensor) contribute to rapid growth of tumor and are emerging hallmarks of cancer. Inhibiting 0 -GIcNAcylation could be a promising anticancer strategy. The gluconeogenic enzyme phosphoenolpyruvate carboxykinase 1 (PCK1) is downregulated in hepatocellular carcinoma (HCC). However, little is known about the potential role of PCK1 in enhanced HBP activity and HCC carcinogenesis under glucose-limited conditions. In this study, PCK1 knockout markedly enhanced the global O-GIcNAcylation levels under low-glucose conditions. Mechanistically, metabolic reprogramming in PCK1-loss hepatoma cells led to oxaloacetate accumulation and increased de novo uridine triphosphate synthesis contributing to uridine diphosphate- $N$-acetylglucosamine (UDP-CIcNAc) biosynthesis. Meanwhile, deletion of PCK1 also resulted in AMPK-GFAT1 axis inactivation, promoting UDP-GIcNAc synthesis for elevated 0 GIcNAcylation. Notably, lower expression of PCK1 promoted CHK2 threonine 378 O-GIcNAcylation, counteracting its stability and dimer formation, increasing CHK2-dependent Rb phosphorylation and HCC cell proliferation. Moreover, aminooxyacetic acid hemihydrochloride and 6-diazo-5-0xo-L-norleucine blocked HBP-mediated O-CIcNAcylation and suppressed tumor progression in liver-specific Pck1-knockout mice. We reveal a link between PCK1 depletion and hyper-0-GIcNAcylation that underlies HCC oncogenesis and suggest therapeutic targets for HCC that act by inhibiting O-CIcNAcylation.
\end{abstract}

\section{Introduction}

Gaining insight into the fundamental role of metabolic reprogramming in cancer has contributed immensely to our understanding of tumorigenesis and cancer progression (1). Nutrient limitations (such as glucose deprivation) in solid tumors may require cancer cells to exhibit the metabolic flexibility required to sustain proliferation and survival $(2,3)$. Increased aerobic glycolysis (the Warburg effect) is one of the main characteristics of cancer cells that supports their intensive growth and proliferation (4). Gluconeogenesis (the pathway opposite to glycolysis) operates during starvation and occurs mainly in the liver, and also plays key roles in metabolic reprogramming, cancer cell plasticity, and tumor growth $(5,6)$. Several key gluconeogenic enzymes, such as phosphoenolpyruvate carboxykinase 1 (PCK1, also known as PEPCK-C), fructose-1,6-bisphosphatase, and glucose-6-phosphatase, were previously found to be dysregulated in several types of cancer, including hepatocellular carcinoma (HCC) (7).

Authorship note: JX, CC, RL, and DC contributed equally to this work. Conflict of interest: The authors have declared that no conflict of interest exists. Copyright: () 2021, American Society for Clinical Investigation.

Submitted: September 30, 2020; Accepted: March 3, 2021; Published: April 15, 2021 Reference information: J Clin Invest. 2021;131(8):e144703.

https://doi.org/10.1172/JCl144703.
The cytoplasmic isoform of PCK1, the first rate-limiting enzyme in hepatic gluconeogenesis, catalyzes the conversion of oxaloacetate (OAA) to phosphoenolpyruvate (PEP). The oncogenic or tumor suppressor roles of PCK1 in different types of human cancers are rather controversial. PCK1 has antitumorigenic effects in gluconeogenic organs (liver and kidney), but has tumor-promoting effects in cancers arising from nongluconeogenic organs (5). In colon-derived tumor cells, PCK1 is hijacked to participate in truncated gluconeogenesis to meet biosynthetic and anabolic needs (8). However, the underlying mechanism determining its aberrant expression and altered function in multiple types of tumors remains incompletely understood.

Recent findings emphasize the role of the hexosamine-biosynthesis pathway (HBP), a subbranch of glucose metabolism, in carcinogenesis $(9,10)$. The HBP and glycolysis share the first 2 steps and diverge at fructose-6-phosphate (F6P). Glutamine-fructose-6-phosphate aminotransferase 1 (GFAT1), the rate-limiting enzyme of the HBP, converts F6P and glutamine to glucosamine-6-phosphate and glutamate. Uridine diphosphate $\mathrm{N}$-acetylglucosamine (UDP-GlcNAc), the end products of HBP, is a donor substrate for $O$-linked $\beta$ - $N$-acetylglucosamine $(O-G l c-$ NAc) modification (also known as $O$-GlcNAcylation) (11). O-GlcNAc transferase-mediated (OGT-mediated) protein $O$-GlcNAcylation is highly dependent on the intracellular concentration of the 


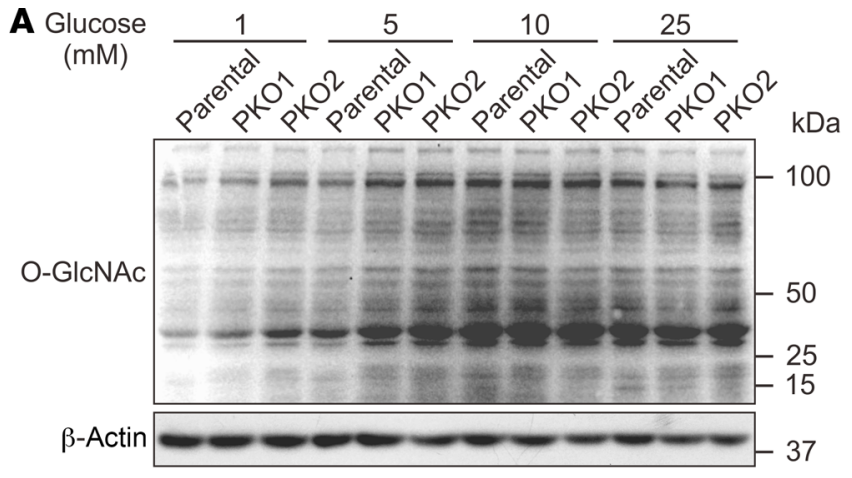

C

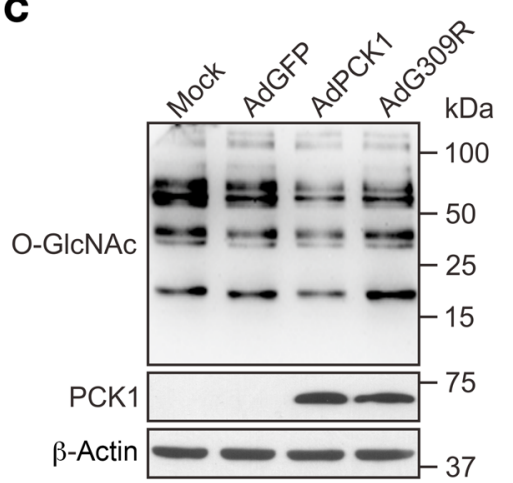

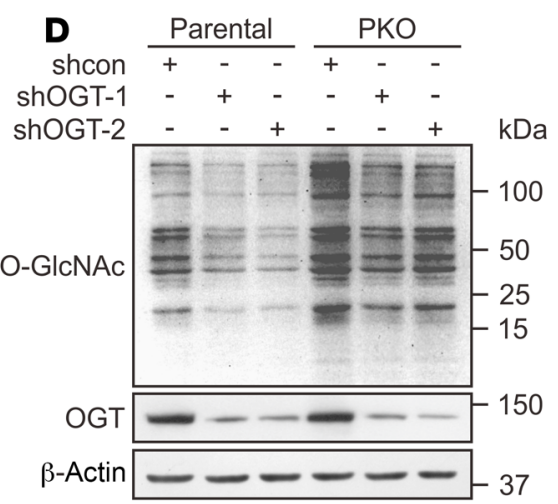

G

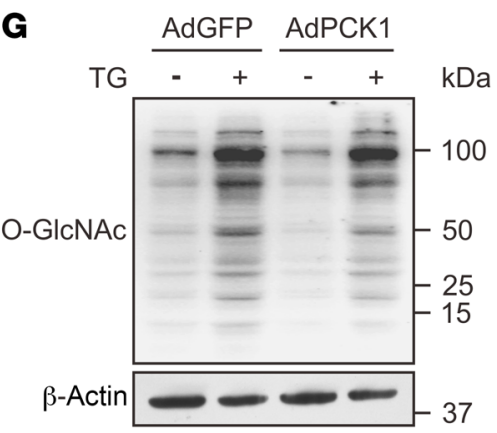

B

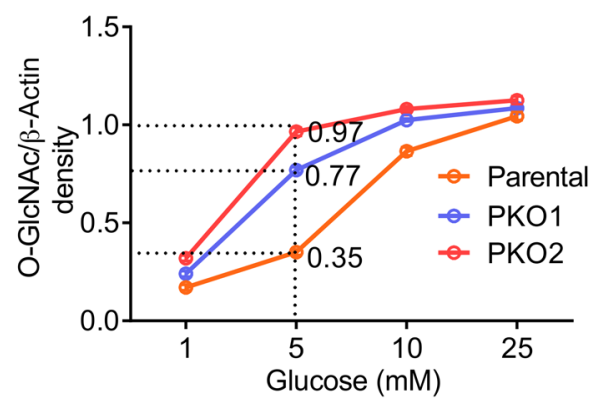

\section{E}

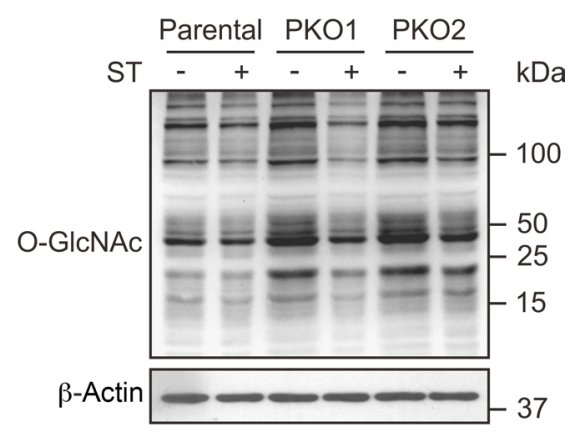

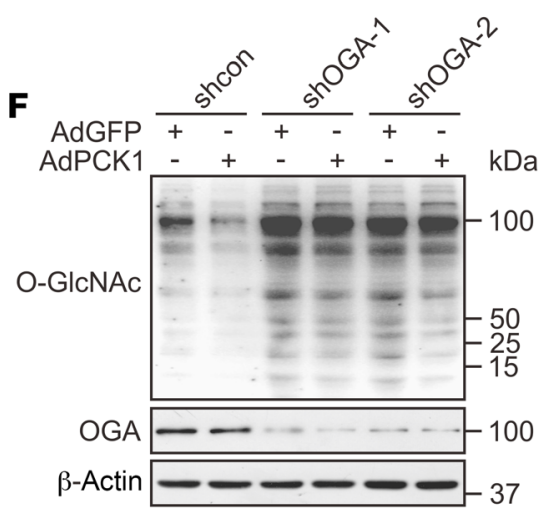
$-37$

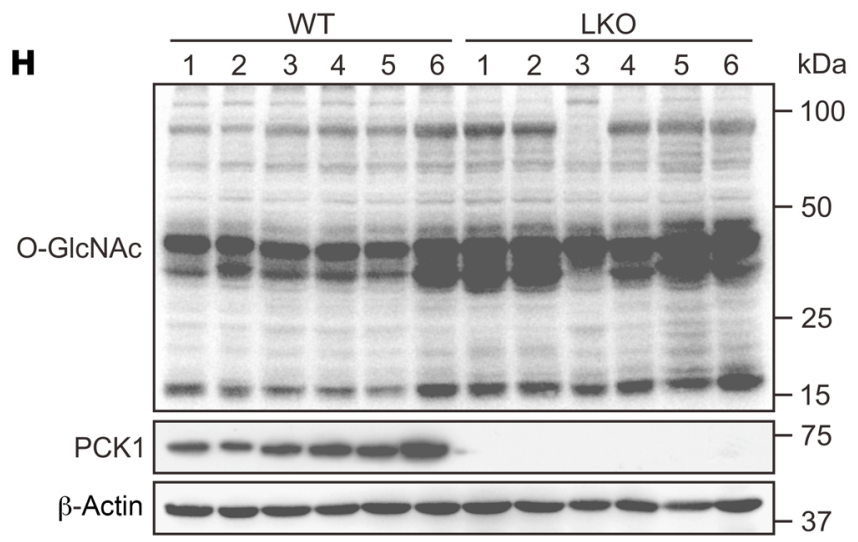

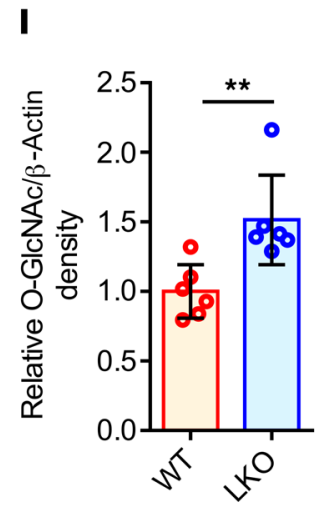

Figure 1. PCK1 deficiency enhances protein $\mathbf{0}$-GIcNAcylation. Immunoblotting analysis of global $O$-GIcNAcylation levels in PCK1-knockout PLC/PRF/5 cells (PKO cells) treated with medium containing different levels of glucose for 12 hours (A). Densitometric analysis was performed with Image-Pro Plus software (B). (C) Representative Western blot analysis of the indicated proteins in SK-Hep1 cells overexpressing green fluorescence protein (GFP; control cells), WT PCK1, or an enzymatically deficient mutant (PCK1 G309R) after incubation in medium containing $5 \mathrm{mM}$ glucose for 12 hours. Mock-treated cells served as a blank control. To determine OGT and protein O-GIcNAcylation levels in PKO cells, cells were transfected with shRNA targeting OCT mRNA or a scrambled control shRNA (shcon) for 48 hours (D), or treated with $50 \mu \mathrm{M}$ ST (ST045849, OGT inhibitor) for 12 hours (E). For immunoblotting analysis of SK-Hep1 cells, PCK1-expressing cells were transfected with an OGA shRNA1/2 plasmid for 48 hours (F), or treated with $25 \mu \mathrm{M}$ TG (Thiamet G, OCA inhibitor) for 12 hours (G). Immunoblotting $(\mathbf{H})$ and densitometric analysis (I) of liver tumors from DEN/CCl -induced WT and LKO mice after fasting for 12 hours. Data are mean $\pm S D$ ( $n=6$ experiments). ${ }^{* *} P<0.01$, 2-tailed unpaired Student's $t$ test. 
donor substrate UDP-GlcNAc, which is proposed to be a nutrient sensor that couples metabolic and signaling pathways $(12,13)$. Increased glucose flux through the HBP and elevated UDP-GlcNAc contribute to hyper-O-GlcNAcylation in cancer cells (14). Previous data suggest that elevated $O$-GlcNAcylation may serve as a hallmark of cancer (15).

Similar to phosphorylation, O-GlcNAcylation is a dynamic posttranslational modification that regulates protein subcellular localization, stability, protein-protein interactions, or enzymatic activity according to the nutrient demands of cells (16). OGT and $O$-GlcNAcase (OGA) are the only enzymes known to be responsible for adding and removing $N$-acetylglucosamine (GlcNAc) on serine and threonine residues of target proteins (17). Numerous oncogenic factors, including c-MYC, Snail, and $\beta$-catenin, are targets of $O$-GlcNAcylation (18-20). Therefore, modulating the HBP or $O$-GlcNAcylation (which regulate oncogenic activation in human cancers) represents a promising anticancer strategy that can potentially be used in combination with other treatments $(21,22)$.

In this study, we explored the role of the gluconeogenic enzyme PCK1 in regulating the HBP and HCC proliferation under low-glucose conditions. We unravel a molecular mechanism responsible for enhanced UDP-GlcNAc biosynthesis and O-GlcNAcylation induced by PCK1 depletion, and delineate the functional importance of checkpoint kinase 2 (CHK2) O-GlcNAcylation in HCC tumorigenesis. Importantly, our study reveals a novel link between the gluconeogenic enzyme PCK1 and HBP-mediated $O$-GlcNAc modification, which suggests a therapeutic strategy for treating HCC.

\section{Results}

PCK1 deficiency increases global O-GlcNAcylation in hepatoma cells under low-glucose conditions and promotes HCC proliferation. To explore the role of the gluconeogenic enzyme PCK1 in O-GlcNAcylation, we first analyzed the global $O$-GlcNAcylation levels of hepatoma cells in response to PCK1 modulation with various concentrations of glucose ( 1 to $25 \mathrm{mM}, 12$ hours). In the presence of $5 \mathrm{mM}$ glucose, PCK1 knockout markedly elevated the global $O$-GlcNAcylation levels (Figure 1, A and B), whereas overexpression of WT PCK1 markedly decreased the global $O$-GlcNAcylation levels in SK-Hep1, Huh7, and MHCC-97H cells (Figure 1C and Supplemental Figure 1, A-C). However, the global O-GlcNAcylation in the tumor cells derived from nongluconeogenic organs did not obviously change upon PCK1 overexpression in vitro (Supplemental Figure 1D). Interestingly, the catalytically inactive G309R mutant of PCK1 (23) was unable to reduce the $O$-GlcNAcylation levels in these hepatoma cells under the same culture conditions (Figure 1C and Supplemental Figure 1, B and C), suggesting that the enzymatic activity of PCK1 may play an attenuating role in regulating cellular $O$-GlcNAcylation levels. In addition, this observation was confirmed via pharmacological or transcriptional inhibition of OGT and OGA (Figure 1, D-G), suggesting PCK1 inhibits OGT-mediated $O$-GlcNAcylation. Interestingly, PCK1 did not change the mRNA or protein expression levels of OGT, OGA, and GFAT1, the key enzymes involved in regulating $O$-GlcNAcylation and HBP (Supplemental Figure 1, E-N). In addition, our cell-proliferation assays indicated that PCK1 suppresses hepatoma cell proliferation, depending on its enzymatic activity and the cellular $O$-GlcNAcylation levels (Supplemental Figure 2).
Additionally, we used an $N$-nitrosodiethylamine (DEN) and $\mathrm{CCl}_{4}$-induced mouse HCC model to verify the above results in vivo. The $O$-GlcNAcylation levels in the hepatic tumors of liver-specific Pck1 knockout (LKO) mice were significantly higher than those in WT mice after 12 hours of fasting (Figure 1, H and I). Furthermore, using an orthotopic HCC mouse model, we found that PCK1 overexpression decreased the $O$-GlcNAcylation levels and inhibited HCC growth (Supplemental Figure 3). Together, these data demonstrated that PCK1 decreases the global O-GlcNAcylation levels in HCC under a low-glucose condition and suppresses hepatoma cell proliferation, both in vitro and in vivo. The enzymatic activity of PCK1 is indispensable for its tumor suppressor role in HCC.

PCK1 deficiency promotes UDP-GlcNAc biosynthesis via oxaloacetate accumulation. Next, a metabolomics assay was performed with AdPCK1- and AdGFP-infected SK-Hep1 cells to explore metabolic changes occurring after PCK1 overexpression (PCK1-OE) under a low glucose concentration $(5 \mathrm{mM})$. Principal component analysis showed that PCK1 overexpression dramatically changed the intracellular metabolic profile of SK-Hep1 cells (Supplemental Figure 4A). Levels of several metabolites of the HBP decreased after PCK1 overexpression, including fructose 6-phosphate, $\mathrm{N}$-acetyl glucosamine 1-phosphate (GlcNAc-1-P), and UDP-GlcNAc (the HBP end product), as shown in Figure 2, A and B, and Supplemental Figure 4B. Our targeted liquid chromatography-tandem MS (LC-MS/MS) results showed that UDP-GlcNAc significantly decreased in PCK1-OE SK-Hep1 cells (Figure 2C), but increased in PCK1-KO (PKO) cells (Figure 2D), suggesting that PCK1 may negatively regulate UDP-GlcNAc biosynthesis for $O$-GlcNAcylation. To investigate how PCK1 modulates UDP-GlcNAc biosynthesis, we performed pathway-enrichment analysis of metabolite profiles and found that several metabolic pathways were significantly affected, including purine and pyrimidine metabolism, which is required for uridine triphosphate (UTP) synthesis (Supplemental Figure 4C). Since the HBP uses glucose, glutamine, acetyl-CoA, and UTP to produce the amino sugar UDP-GlcNAc (Figure 2E), we assumed that PCK1 may regulate UDP-GlcNAc biosynthesis partially via UTP synthesis. Indeed, metabolomics data showed that levels of several metabolites involved in UTP synthesis, including Asp (aspartate, critical metabolite in de novo UTP synthesis), declined upon PCK1 overexpression (Figure 2F), besides metabolites in glycolysis and the tricarboxylic acid (TCA) cycle (Figure 2A and Supplemental Figure 4, D-G), suggesting that PCK1 may play role in TCA cataplerosis and UTP synthesis which contribute to UDP-GlcNAc biosynthesis.

PCK1 catalyzes the conversion of OAA (an intermediate of the TCA cycle) to PEP. Consistent with the results of a previous study (8), restoring PCK1 decreased the OAA concentration in SK-Hep1 cells (Figure 2G). Considering that OAA is converted to Asp by the mitochondrial glutamate-oxaloacetate transaminase (GOT2, also known as aspartate aminotransferase 2; Supplemental Figure $4 \mathrm{H}$ ), we speculated that PCK1 repression may promote UDP-GlcNAc biosynthesis through Asp conservation caused by OAA accumulation. As expected, adding OAA strengthened UDP-GlcNAc biosynthesis (Figure 2H). To acquire a definitive proof of UDP-GlcNAc synthesis from OAA accumulation, we used a stable isotopic tracing approach to detect dynamic metabolic flux. The relative 


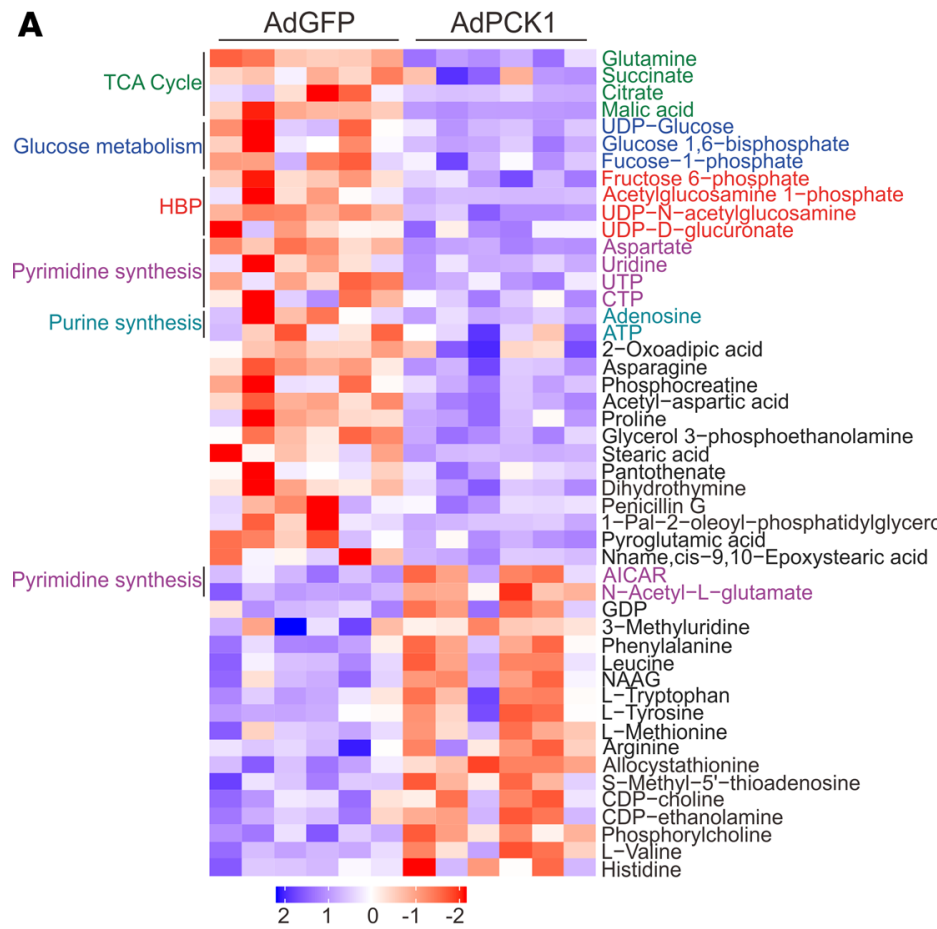

$\mathbf{F}$

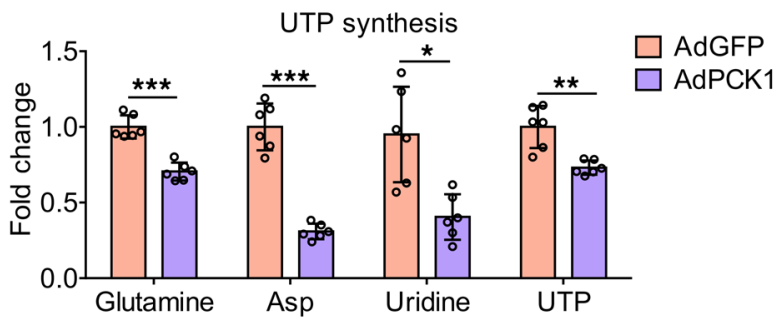

UDP-GICNAC

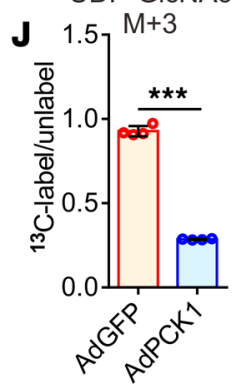

B

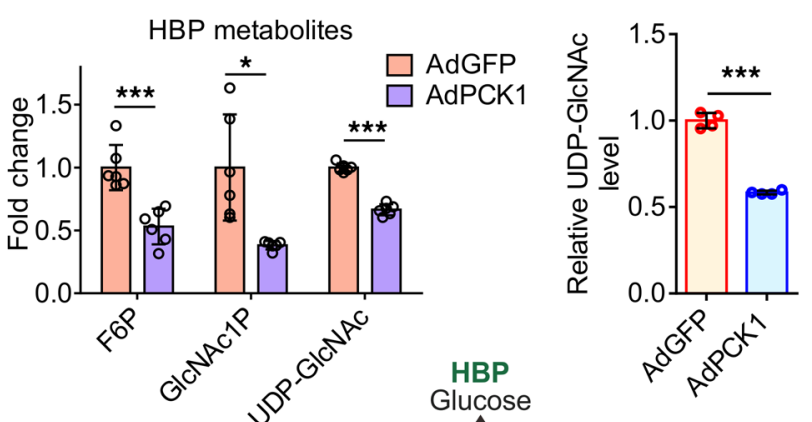

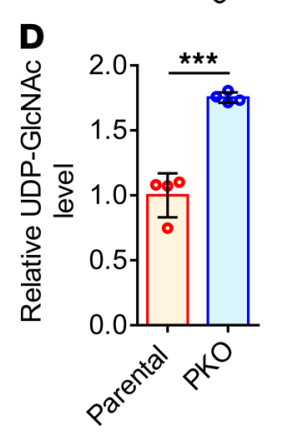

G
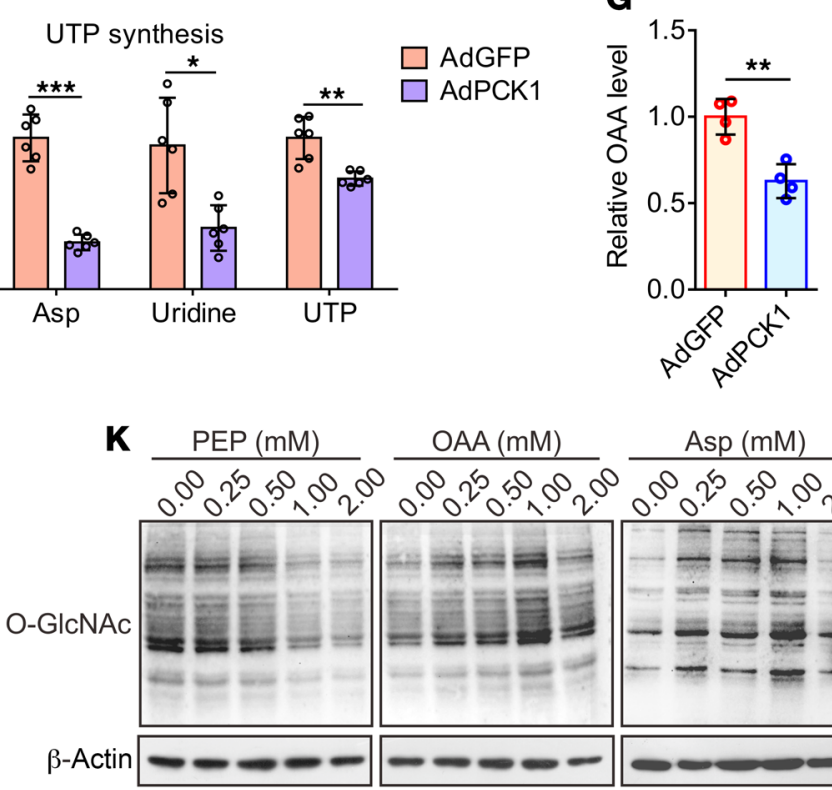

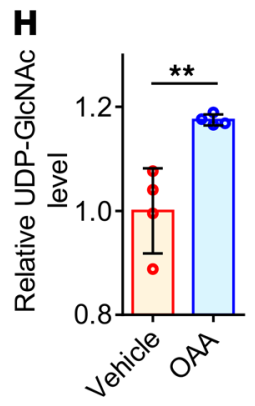

E $\quad \begin{aligned} & \downarrow \\ & F 6 P\end{aligned}$

GFAT1) $\downarrow$ Glutamine Asp

GICN6P

$\downarrow$ Ac-CoA Pyrimidine

GIcNAc6P

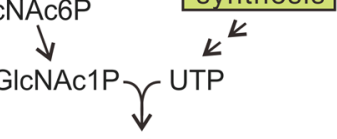

UDP-GIcNAc

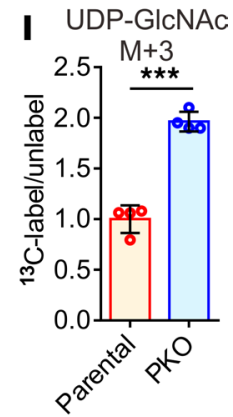

$\mathbf{L}$

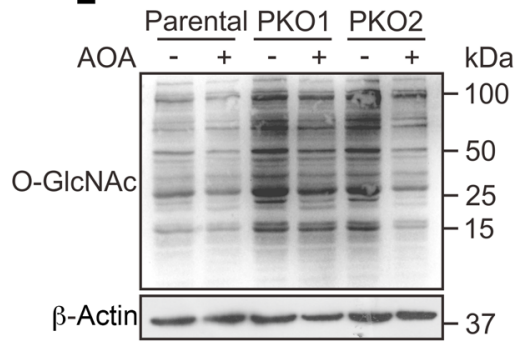

$\beta$-Actin -37
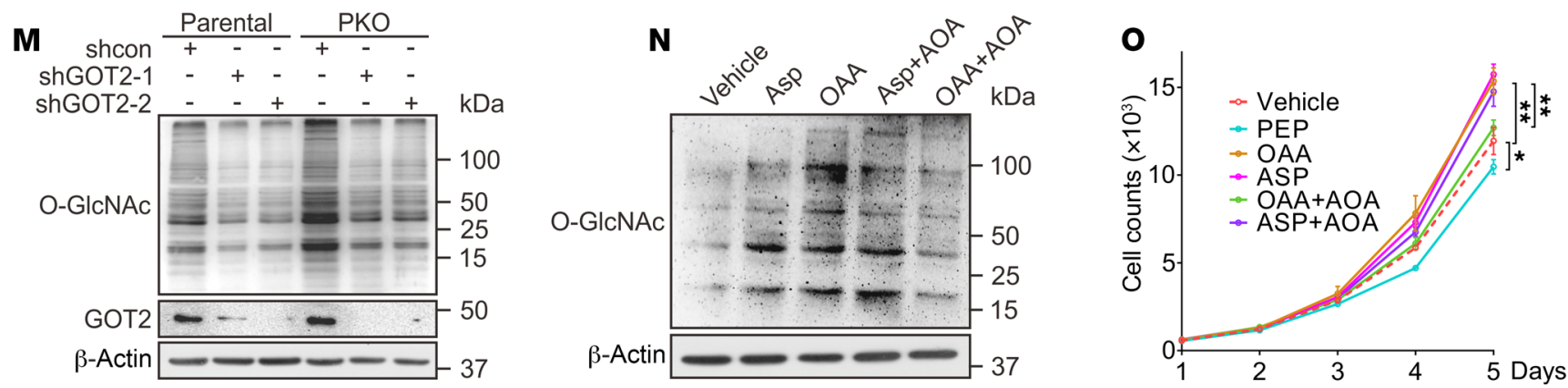
Figure 2. PCK1 knockout promotes UDP-GICNAc synthesis partially through oxaloacetate accumulation. Heatmap of metabolites $(\mathbf{A})$ and fold-changes in intermediate metabolites of the HBP (B). Relative UDP-GIcNAc levels were measured by LC-MS in PCK1-OE SK-Hep1 cells (C) and PKO cells (D). (E) Schematic representation of the HBP. Glucose intake feed into the HBP that produces UDP-GIcNAc. N-Acetylglucosamine-1-phosphate (GICNAc1P) and UTP, terminal metabolites of the $\mathrm{HBP}$ and pyrimidine synthesis, represent the final rate-limiting steps of UDP-GIcNAc synthesis. (F) Fold-changes in the intermediate metabolites of uridine synthesis. (G) Relative OAA levels, as measured by LC-MS in PCK1 -OE SK-Hep1 cells. (H) Relative levels of UDP-GIcNAc, as measured by LC-MS in PKO cells treated with $1 \mathrm{mM} O A A . \mathrm{m}+3$ labeled UDP-GIcNAc levels in PKO cells (I) and PCK1 overexpressing SK-Hep1 cells (J) cultured with ${ }^{13} \mathrm{C}_{5}$-glutamine. (K) Protein $\mathrm{O}$-GlcNAcylation levels in SK-Hep1 cells cultured for 12 hours in medium containing $5 \mathrm{mM}$ glucose and PEP (left), OAA (middle), or Asp (right). Protein O-GlcNAcylation levels in PKO-cells treated with $20 \mu \mathrm{M}$ AOA for 12 hours (L) or transfected with a GOT2 shRNA1/2 plasmid for 48 hours (M). (N) Immunoblots of SK-Hep1 lysates treated for 12 hours with OAA (1 mM), Asp (1 mM), or AOA $(20 \mu \mathrm{M})$, as indicated. (0) Proliferation ability of SK-Hep1 cells treated as indicated. Data are mean \pm SD ( $n \geq 3$ experiments). ${ }^{*} P<0.05$, ${ }^{* *} P<0.01,{ }^{* *} P<0.001,2$-tailed Student's $t$ test (2 groups) or 1-way ANOVA followed by Tukey's test (more than 2 groups). Data are representative of at least 3 independent experiments.

abundance of M+3 UDP-GlcNAc was significantly increased in PKO cells when incubated with ${ }^{13} \mathrm{C}_{5}$-glutamine, whereas it decreased in PCK1-OE cells (Figure 2, I and J). The isotopomers of the metabolites involved in the TCA cycle including OAA, and UTP de novo synthesis were reduced in the PCK1-OE cells, meanwhile Asp was increased in PKO cells (Supplemental Figure 5), suggesting that PCK1 deficiency strongly boosts the entry of OAA into UDP-GlcNAc synthesis.

Accordingly, both OAA and Asp enhanced $O$-GlcNAcylation in SK-Hep1 cells, while high concentrations of PEP (above $0.5 \mathrm{mM}$ ) showed an opposite effect (Figure 2K). Next, we tested whether GOT2 can contribute to HBP-mediated $O$-GlcNAcylation in PKO cells. We found that treatment with aminooxyacetic acid (AOA), a specific inhibitor of GOT2, decreased the UDP-GlcNAc levels in PKO cells (Supplemental Figure 4I). Moreover, AOA treatment or short-hairpin RNA-mediated (shRNA-mediated) GOT2 knockdown reduced the O-GlcNAcylation levels in PKO cells (Figure 2, $\mathrm{L}$ and $\mathrm{M}$ ). In addition, AOA markedly blocked $O$-GlcNAcylation induced by OAA treatment, but failed to moderate the effect of Asp, indicating that GOT2 plays an essential role in the metabolism of OAA converted to Asp, the HBP, and tumorigenesis (Figure 2, $\mathrm{N}$ and $\mathrm{O}$ and Supplemental Figure 4J). Taken together, these data provide strong evidence supporting the contributions of OAA accumulation and the GOT2-mediated pathway to enhanced UDP-GlcNAc biosynthesis and hyper-O-GlcNAcylation in $\mathrm{PKO}$ cells.

Restoration of PCK1 suppresses O-GlcNAcylation by activating the AMPK-GFAT1 axis. The final rate-limiting step of UDP-GlcNAc synthesis involves UTP and GlcNAc-1-P (Figure 2E). Our metabolomics analysis showed that both UTP and GlcNAc-1-P levels were significantly decreased in PCK1-OE cells (Figure 2, B and F). Since OAA accumulation contributes to the downstream UTP increase and GFAT1 is the rate-limiting enzyme in GlcNAc-1-P synthesis, we explored whether GFAT1 activity also regulates UDP-GlcNAc production. Previously, we reported that PCK1 activates AMP-activated protein kinase (AMPK) upon glucose deprivation in HCC (24). Oth- er groups showed that AMPK activation reduces $O$-GlcNAcylation through GFAT1 phosphorylation (which diminished GFAT1 activity) in endothelial cells and cardiac hypertrophy $(25,26)$. We speculated that PCK1 may also inhibit UDP-GlcNAc synthesis through the AMPK-GFAT1 axis. Thus, we tested whether PCK1 can suppress the HBP through the AMPK-GFAT1 axis under low-glucose conditions. As expected, PCK1 overexpression promoted the phosphorylation of both AMPK and GFAT1 (Figure 3A), whereas PCK1-KO downregulated $\mathrm{p}-\mathrm{AMPK}$ and $\mathrm{p}-\mathrm{GFAT1}$ production (Figure $3 \mathrm{~B}$ ). The AMPK activator metformin partially offset hyper-O-GlcNAcylation mediated by PCK1 depletion (Figure 3C). However, shRNA-mediated knockdown of AMPK in PCK1-OE cells rescued the inhibitory effects of PCK1 on $O$-GlcNAcylation (Figure 3D).

Furthermore, we investigated whether the inhibition of hepatoma cell proliferation in response to PCK1 depended on the AMPK-GFAT1 axis. We found that metformin suppressed PKO cell proliferation (Figure 3, E and F). In contrast, shRNA against AMPK mRNA (shAMPK) promoted PCK-OE cell proliferation (Figure 3, G and H). In addition, the GFAT1 inhibitor 6-diazo-5oxo- $L$-norleucine (DON) reduced UDP-GlcNAc biosynthesis (Figure 3I), O-GlcNAcylation levels (Figure 3J), and PKO cell proliferation (Figure 3, E and F). These data indicate that PCK1 suppresses HBP-mediated $O$-GlcNAcylation and HCC proliferation partially via activation of the AMPK-GFAT1 axis. PCK1 deficiency boosts flux through the HBP and results in an increased availability of UDP-GlcNAc for $O$-GlcNAcylation. Therefore, both OAA accumulation and the AMPK-GFAT1 axis contributed to hyper-O-GlcNAcylation and PKO cell proliferation upon glucose deprivation (Figure 3K).

OGT mediates CHK2 O-GlcNAcylation in PCK1-deficient hepatoma cells. To further explore how OGT-mediated protein $O$-GlcNAcylation facilitates hepatoma cell proliferation in PKO cells, we used immunoprecipitation coupled with tandem MS (IP-MS/MS) to screen for proteins that specifically interact with OGT (Figure 4A and Supplemental Figure 6A). Flag-tagged OGT was transiently expressed in PKO cells, and subsequent IP-MS identified 618 candidate OGT-binding proteins (Supplemental Table 1). Pathway-enrichment analysis indicated that several proteins were involved in metabolic processes, apoptotic processes, and cell-cycle progression (Figure 4B). We then focused on $\mathrm{CHK} 2$, which is required for checkpoint-mediated cell-cycle arrest (27). Interactions between OGT and CHK2 were confirmed by coimmunoprecipitation (co-IP) experiments in HEK293 cells (Supplemental Figure 6, B and C) and PKO cells (Figure 4, C-E). Confocal analysis also indicated that OGT and CHK2 colocalized in the nucleus (Figure 4F and Supplemental Figure 6D). To define the precise region(s) in CHK2 required for this interaction, we expressed full-length HA-tagged OGT in combination with different Flag-tagged fragments of CHK2 in HEK293 cells (Figure 4G). The C-terminal region of CHK2 (amino acids 69-543) containing kinase domains showed a strong interaction, whereas the $\mathrm{N}$-terminal region (amino acids 1-175) did not interact with OGT (Figure 4H).

Then, we determined whether CHK2 can be modified via $O$-GlcNAc. Immunoprecipitated, Flag-tagged CHK2 exhibited a strong O-GlcNAc signal in HKE293 cells (Figure 4I). Endogenous CHK2 O-GlcNAcylation was confirmed by affinity chroma- 
A

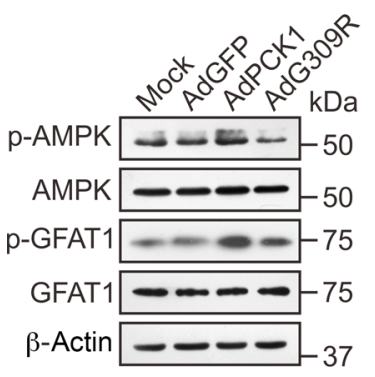

B

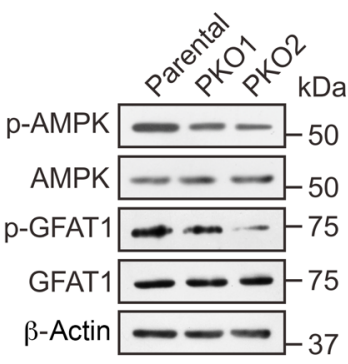

C

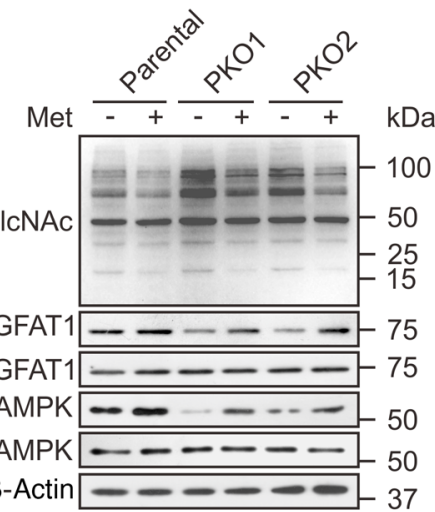

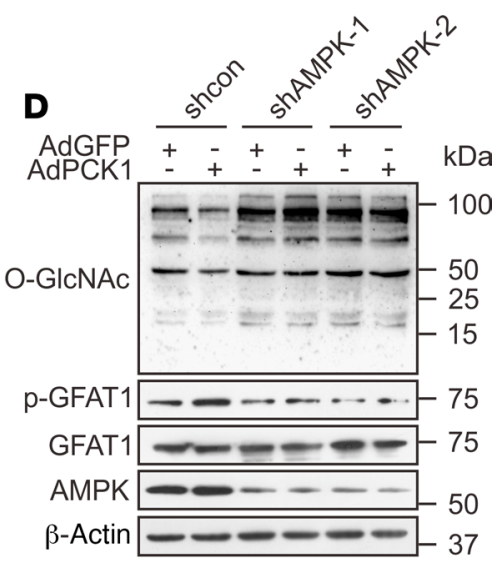
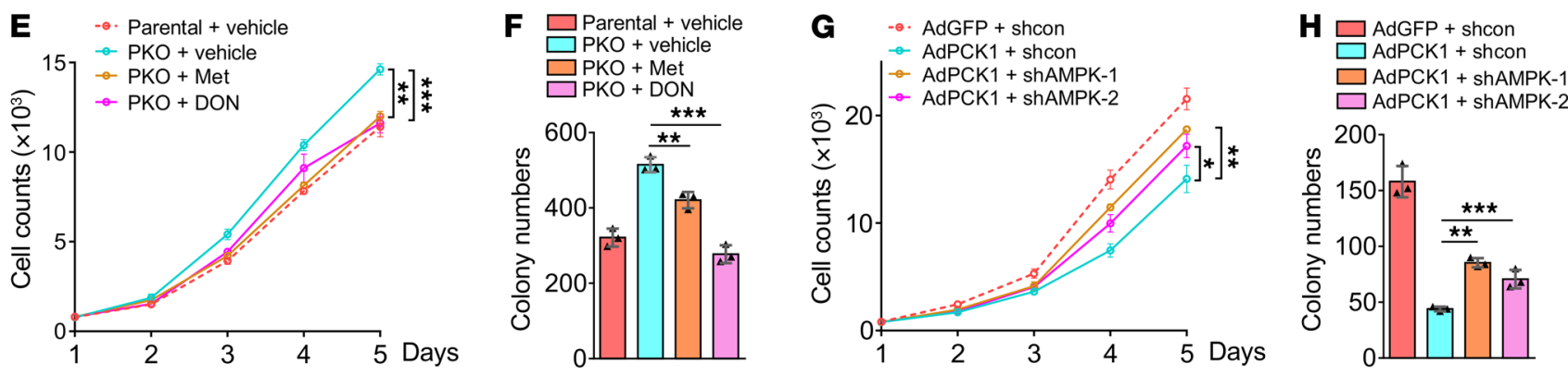

I

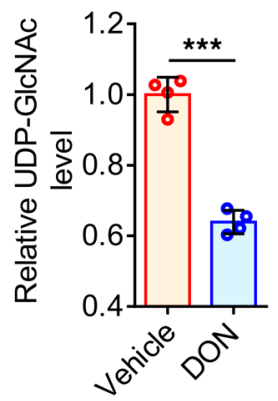

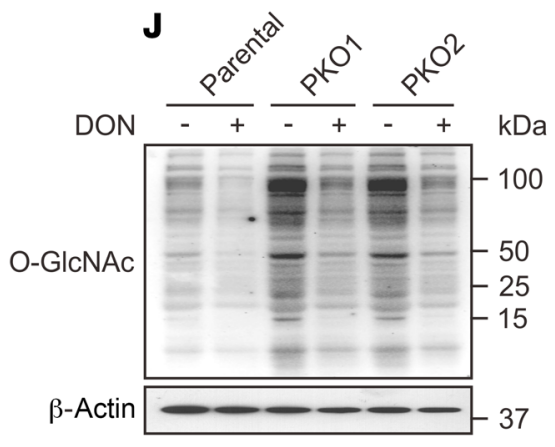

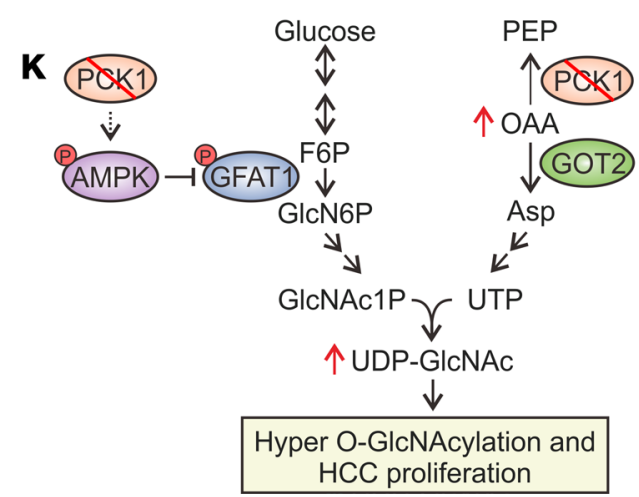

Figure 3. PCK1 activates AMPK ${ }^{\text {Thr72 }} /$ CFAT1 $^{\text {Ser243 }}$ phosphorylation and inhibits UDP-GIcNAc biosynthesis. Representative immunoblots showing AMPK ${ }^{\text {Thr172 }}$ and GFAT1 ${ }^{\text {Ser243 }}$ phosphorylation in PCK1-OE cells (A) and PKO cells (B). For immunoblot analysis, PKO cells were treated with metformin (Met, 2 mM) for 12 hours (C) or PCK1-OE cells were transfected with an AMPK shRNA1/2 plasmid (D). (E-H) Hepatoma cell growth curves and colony formation capacity. PKO cells and PCK1-OE cells were treated as indicated. (I) Relative levels of UDP-GIcNAc in PKO cells treated for 24 hours with the GFAT1 inhibitor DON (20 $\mu \mathrm{M})$, as measured by LC-MS. (J) PKO cells were treated with $20 \mu \mathrm{M}$ DON for 24 hours. (K) Working model whereby PCK1 ablation promotes UDP-CIcNAc biosynthesis, $\mathrm{O}$-GIcNAcylation, and proliferation of HCC cells through increased oxaloacetate accumulation and activation of the AMPK-GFAT axis. Data are represented as mean $\pm \mathrm{SD}$ ( $n \geq 3$ experiments). ${ }^{*} P<0.05$, ${ }^{* *} P<0.01$, ${ }^{* *} P<0.001$, as determined using 2-tailed unpaired Student's $t$ test (2 groups) or 1 -way ANOVA, followed by Tukey's test (more than 2 groups). Data are representative of at least 3 independent experiments.

tography in the presence of the OGA inhibitor PUGNAc, using succinylated wheat germ agglutinin (sWGA), a modified lectin that specifically binds $O$-GlcNAc-containing proteins (Figure 4J). In addition, PCK1-OE and ST (ST045849, the OGT inhibitor) decreased CHK2 O-GlcNAcylation, while PUGNAc (the OGA inhibitor) partially reversed its level (Figure $4, \mathrm{~K}$ and $\mathrm{L}$ ). In contrast, PCK1-KO or OAA treatment strengthened CHK2 O-GlcNAcylation under low-glucose conditions (Figure 4, $\mathrm{L}$ and $\mathrm{M}$ ).

Next, we sought to map the O-GlcNAcylation site(s) on CHK2. Flag-tagged CHK2 was purified from PKO cells and analyzed by
MS. As shown in Figure 4N, threonine 378 (T378) was the main $O$-GlcNAcylation site on CHK2. We then generated site-specific point mutants of CHK2. Mutating T378 to Ala (T378A) largely reduced the $O$-GlcNAc signal compared with WT CHK2 and the T383A mutant control (Figure $4 \mathrm{O}$ and Supplemental Figure 6E), indicating that $\mathrm{T} 378$ is the major CHK2 site carrying the $\mathrm{O}-\mathrm{Glc}-$ NAc modification. The potentially $O$-GlcNAcylated residue T378 and the surrounding amino acids are highly conserved among vertebrates (Figure 4P), indicating that it serves an evolutionarily conserved role regulating the CHK2 protein. Taken together, these 
data indicate that $\mathrm{CHK} 2$ interacts with and can be $\mathrm{O}$-GlcNAcylated by OGT in PKO cells.

$O$-GlcNAcylation on T378 stabilizes CHK2 and promotes hepatoma cell proliferation. To examine the effect of $O$-GlcNAcylation on CHK2 under the low-glucose conditions, Flag-tagged WT and T378A CHK2 were overexpressed with HA-tagged ubiquitin (HA-Ub) in PCK1-KO and parental PLC/PRF/5 cells. WT CHK2 ubiquitination was alleviated in $\mathrm{PKO}$ cells compared with parental cells, whereas the T378A mutation or OGT inhibitor ST045849 enhanced CHK2 ubiquitination (Figure 5A). We then performed a series of cycloheximide-chase experiments to assess the half-life of these proteins. Endogenous $\mathrm{CHK} 2$ was more stable, with a halflife of more than 24 hours, in PLC/PRF/ 5 cells treated with PUG$\mathrm{NAc}$, indicating $\mathrm{CHK} 2 \mathrm{O}-\mathrm{GlcNAcylation}$ may enhance its stability (Supplemental Figure 7, A and B). In comparison with the parental cells, the CHK2 half-life was prolonged in PKO cells, but the T378A mutation or ST0 45849 treatment reduced CHK2 half-life from 24 hours to 12 hours (Figure 5, B-E). In addition, overexpression of WT PCK1 promoted CHK2 ubiquitination and degradation (Supplemental Figure 7, C-G). As expected, the G309R PCK1 mutation did not affect the half-life of CHK2 (Supplemental Figure 7, D-G). These results suggested that $O$-GlcNAc modification of T378 stabilizes CHK2 by preventing its ubiquitination and degradation in PCK1-deficient hepatoma cells.

Given that CHK2 dimerization is essential for its activation (28), we next detected CHK2 dimerization in cells cotransfected with vectors encoding Flag-CHK2 and Myc-CHK2. Our results indicated that $\mathrm{PKO}$ cells displayed strengthened $\mathrm{CHK} 2$ dimer formation, whereas ST0 45849 treatment or CHK2 T378A mutant weakened this association (Supplemental Figure 7, $\mathrm{H}$ and I). A similar result was observed by crosslinking analysis (Supplemental Figure 7J), suggesting $O$-GlcNAcylation of CHK2 may promote its dimerization. Interestingly, T378, the autophosphorylation site of $\mathrm{CHK} 2$, is located in the dimerization interface (28). We then performed dimeric CHK2 homology modeling, followed by molecular dynamic (MD) simulation. Our model disclosed that the $O$-GlcNAcylated residue T378 interacts with the amino acid VSLK of another CHK2 kinase domain. The acetylglucosamine group occupies a cavity located in the edge of interaction interface and forms 3 hydrogen bonds with the backbone of VSLK motif, thus might strengthen the stability of the CHK2 dimer (Supplemental Figure 7, K-M). Since dimerization promotes CHK2 activation and phosphorylates its downstream targets, such as retinoblastoma (Rb) in HCC (29), we subsequently checked the phosphorylation of CHK2 substrates and downstream signaling in response to PCK1 expression. Overexpressing WT PCK1 decreased the p-Rb and p-CDK2 levels, but increased the p27 levels (Figure 5F). In contrast, PCK1 KO or OAA treatment reversed the regulatory effects of these molecules (Figure 5, G and H). Notably, the OGT inhibitor ST045849 or the GFAT1 inhibitor DON partially offset the regulatory effects mediated by PCK1 deficiency (Figure 5, G and I). These data indicated that $\mathrm{O}$-GlcNAcylation promotes $\mathrm{CHK} 2$ dimerization and subsequently enhances downstream $\mathrm{Rb}$ phosphorylation.

To further test whether the loss of CHK2 O-GlcNAcylation affects its downstream signaling and hepatoma cell proliferation, we transiently overexpressed WT CHK2 or the T378A mutant in
CHK2-KO cells. WT CHK2 restored the phosphorylation of $\mathrm{Rb}$ and CDK2 and promoted HCC proliferation, whereas the CHK2 T378A mutant failed to exert this stimulatory role on tumorigenesis (Figure 5J and Supplemental Figure 8, A and B), indicating that T378 O-GlcNAcylation plays an essential role in CHK2 activation. The T378A point mutation (which eliminated the $O$-GlcNAc modification) decreased the capacity of CHK2 to phosphorylate $\mathrm{Rb}$. In agreement, the OGA inhibitor PUGNAc enhanced the ability of WT CHK2 to phosphorylate Rb and CDK2, but not that of the CHK2 T378A mutant (Figure 5J). Accordingly, PCK1 $\mathrm{KO}$ or OAA treatment promoted WT CHK2 activation (Figure 5, $\mathrm{K}$ and $\mathrm{L}$ ). Finally, we explored whether PCK1 deficiency-induced malignancy may rely on $\mathrm{CHK} 2 \mathrm{O}$-GlcNAcylation. As expected, CHK2 depletion suppressed hepatoma cell proliferation and G1/S transition induced by PCK1 deficiency, which was rescued by reexpressing WT CHK2, but not the T378 mutant (Supplemental Figure 8, C-H). In addition, CHK2 depletion dramatically reduced the growth of orthotopic tumor bearing PKO PLC/PRF/5 in nude mice (Supplemental Figure 9). These data suggest that CHK2 T378 O-GlcNAcylation conferred a growth advantage for the PKO cells. Collectively, these findings indicate that the $O$-GlcNAcylation of residue T378 stabilizes CHK2 and activates its downstream targets such as $\mathrm{Rb}$, thus promoting PCK1-deficient hepatoma cell proliferation in vitro.

Targeting HBP-meditated O-GlcNAcylation suppresses DEN/ $\mathrm{CCl}_{4}$-induced hepatocarcinogenesis in vivo. Next, we used the DEN/ $\mathrm{CCl}_{4}$-induced mouse model of liver cancer to further verify our results in vivo. Based on our in vitro data, we proposed that blocking the HBP with an inhibitor of GOT2 (AOA) or GFAT1 (DON) could suppress the growth of HCC by reducing $O$-GlcNAcylation. LKO mice (Figure 6, A and B) were generated as previously described (30). The mice were treated with $\mathrm{DEN} / \mathrm{CCl}_{4}$ to induce hepatocarcinoma, which was followed by administering AOA or DON (twice a week) for 16 weeks (Figure 6C and Supplemental Figure 10). The LKO mice exhibited accelerated liver tumorigenesis with increased tumor masses and nodules, and higher serum levels of aspartate aminotransferase (AST) and alanine aminotransferase (ALT) (Figure 6, D-H). Our MS data showed that the OAA and UDP-GlcNAc levels were higher in the liver tumors of LKO mice (Figure 6, I and J), indicating that PCK1 deficiency promotes the HBP in vivo. In contrast, mice treated with AOA or DON exhibited slower tumor growth and a reduced number of tumor nodules compared with untreated LKO mice (Figure 6, D-F). Furthermore, AOA or DON treatment also decreased UDP-GlcNAc and $O$-GlcNAcylation levels in LKO mice (Figure $6, \mathrm{~J}-\mathrm{L}$ ). These data suggested that hyper-O-GlcNAcylation conferred a growth advantage for tumor cells in vivo. Consistent with our in vitro data, the levels of total CHK2, O-GlcNAcylated $\mathrm{CHK}$, and $\mathrm{p}-\mathrm{Rb}$ were significantly enhanced in the liver tumors of LKO mice, and were partially reversed by administering AOA or DON (Figure 6L). In summary, these findings suggested that PCK1 depletion increases susceptibility to $\mathrm{DEN} / \mathrm{CCl}_{4}$-induced carcinogenesis and promotes hepatocarcinogenesis via enhanced CHK2 O-GlcNAcylation; thus, blocking HBP-meditated $O$-GlcNAcylation suppresses HCC in LKO mice.

PCK1 deficiency strengthens CHK2 O-GlcNAcylation in primary human HCC. Finally, we investigated PCK1 expression and glob- 
A

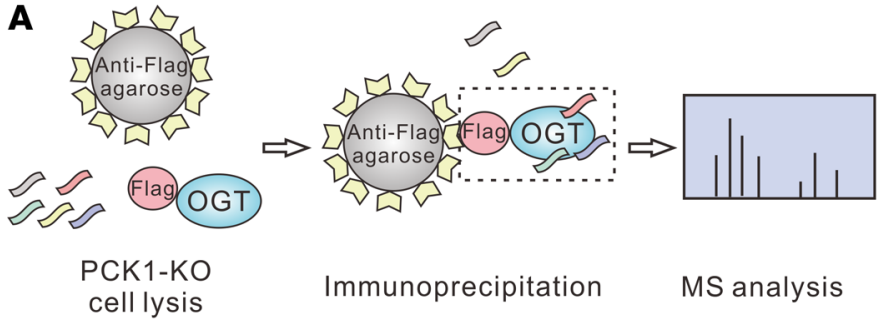

B

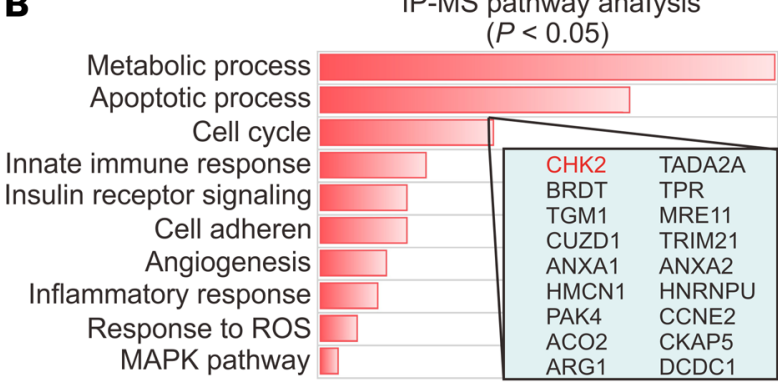

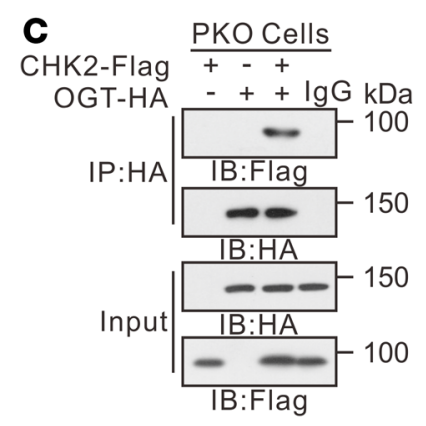

D

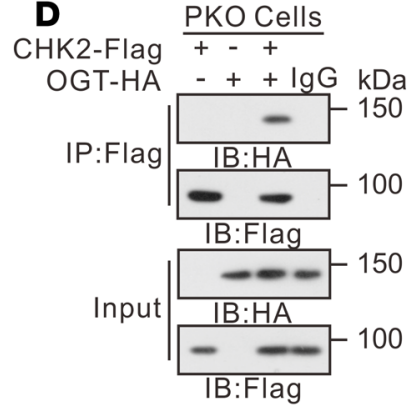

E

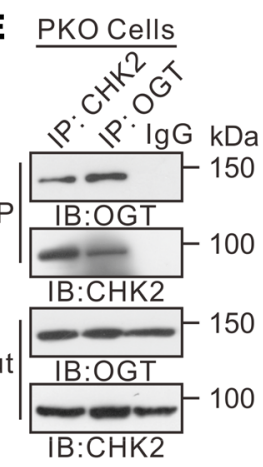

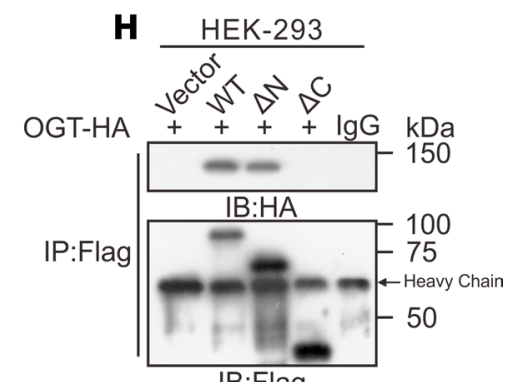

IB:Flag

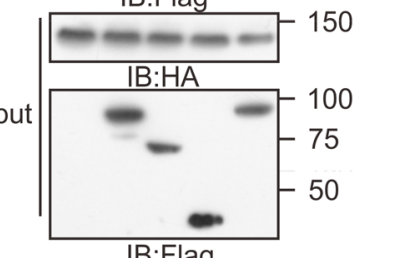

IB:Flag
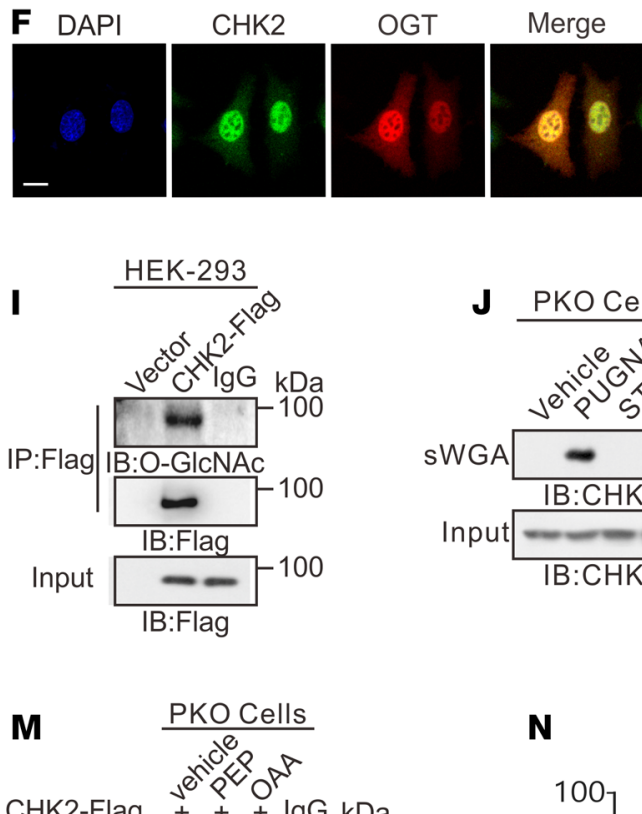

CHK2-Flag $+++\operatorname{lgG~kDa}$

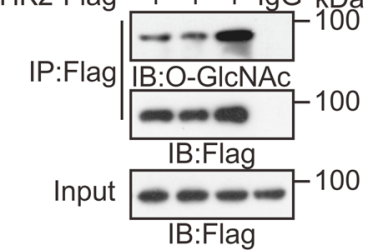

$\mathbf{P}$

$\begin{array}{ll}\text { Frog } & \text { 338 DFGQSKILGETSLMRTLCGT } 358 \\ \text { Chicken } & \text { 345DFGQSKILGETSLMKTLCGT } 365\end{array}$

$\begin{array}{ll}\text { Chicken } & \text { 345DFGQSKILGETSLMKTLCGT } 365 \\ \text { Mouse } & \text { 372DFGQSKILGETSLMRTLCGT } 392\end{array}$

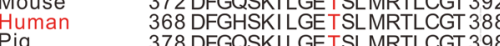

Bovine

Beluga whale 368 DFGQSKILGETSLMRTICGT 388

Bat 356 DFGQSKILGETSLMRTLCGT 376

Mink

Walrus

356 DFGQSKILGETSLMRTLCGT 376

369 DFGQSKILGETSLMRTLCGT 389

368 DFGQSKILGETSLMRTLCGT 388

\section{$\mathbf{N}$}

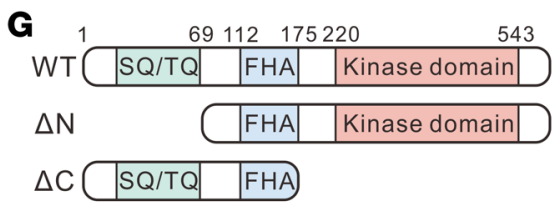

$\underset{\text { PCK1 } 1-H A}{\text { K }} \frac{\text { PKO Cells }}{-++}$

PCK1-HA - + +

$\mathrm{CHK}_{2}$-Flag $+++\operatorname{lgG} \mathrm{kDa}$

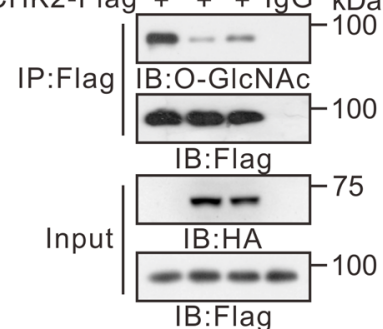

$\mathbf{L}$

PKO Cells
CHK2-Flag $+++\lg G \mathrm{kDa}$

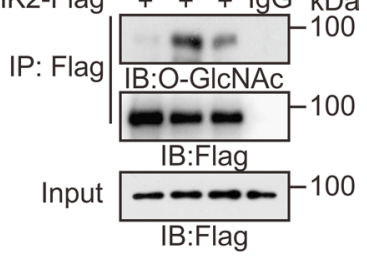

- PKO Cells
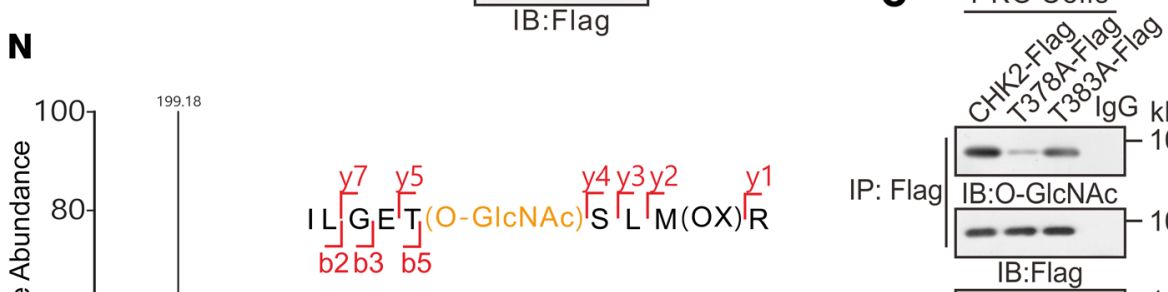

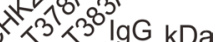

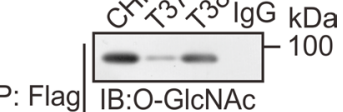

IP: Flag IB:O-GIcNAc
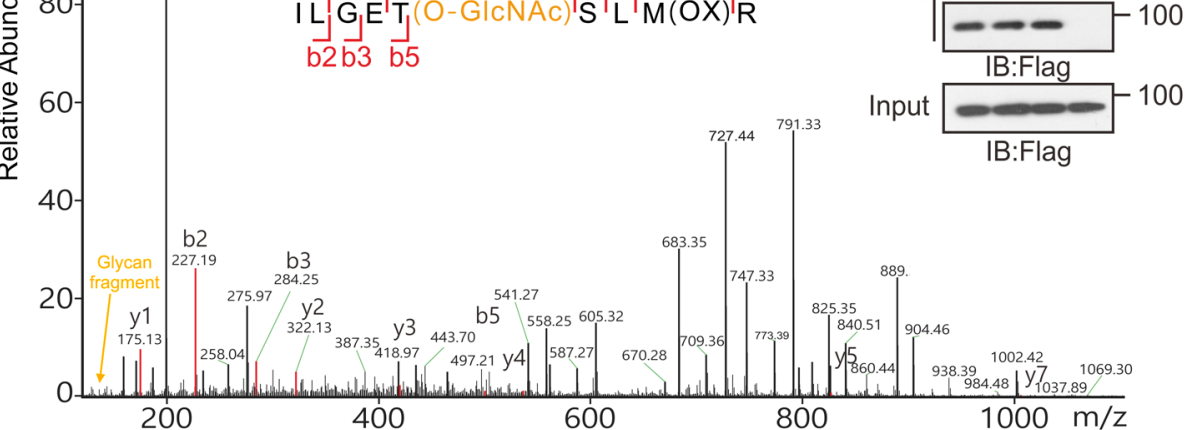
Figure 4. PCK1 deficiency promotes CHK2 O-GIcNAcylation at T378. IP-LC-MS/MS analysis of $O$-GIcNAc-modified proteins, represented by (A) flowchart describing the processes used for IP-LC-MS/MS analysis, and (B) Kyoto Encyclopedia of Genes and Genomes-based analysis of significantly enriched pathways represented by proteins that bound to Flag-tagged OGT. Co-IP of OGT-HA and CHK2-Flag was examined using an anti-HA antibody (C) or an anti-Flag antibody (D) in PKO cells. (E) Co-IP of endogenous OGT and CHK2 in PKO cells. (F) Subcellular colocalization of OCT and CHK2 in SK-Hep1 cell was determined with immunofluorescence staining. Scale bar: $50 \mu \mathrm{m}$. (G) Schematic representation of the CHK2 constructs. WT CHK2 contains 3 domains, including a SQ/TQ cluster domain, a Forkhead-associated (FHA) domain, and a kinase domain. Truncation mutants of CHK2, comprising amino acids (aa) 69-543 or 1-221, were designated as $\Delta \mathrm{N}$ and $\Delta \mathrm{C}$, respectively. $(\mathbf{H})$ Interactions between OGT and full-length $\mathrm{WT}$, the $\Delta \mathrm{N}$, or the $\Delta \mathrm{C}$ in HEK293 cells were determined by co-IP. (I) CHK2 IP with anti-Flag M2 agarose beads in HEK293 cells transfected with CHK2-Flag or a vector control. (J) PKO cells were treated with $50 \mu \mathrm{M}$ PUGNAc or $50 \mu \mathrm{M}$ ST for 24 hours, incubated in $5 \mathrm{mM}$ glucose, and followed by a sWGA pull-down assay. Western blot was determined by anti-CHK2. Cell lysates of PCK1-OE cells (K), PKO cells (L), or SK-Hep1 cells treated with $1 \mathrm{mM}$ PEP or OAA (M) were immunoprecipitated with antiFlag agarose beads and immunoblotted, as indicated. (N) LC-MS analysis of CHK2-Flag identified residue T378 as the CHK2 O-GIcNAcylation site, which corresponded to the 0 -GIcNAcylated CHK2 peptide ILGETSLMR. (0) IP with anti-Flag M2 agarose beads in PKO cells. Cells were transfected with vectors encoding Flag-tagged versions of WT CHK2, T378A CHK2, or T383A CHK2. (P) Cross-species sequence alignment of CHK2.

al $O$-GlcNAcylation in 40 paired human primary HCC tissues and tumor-adjacent normal tissues. As shown by our IHC and immunoblot results, PCK1 was downregulated in most HCC tissues (Figure 7, A-C and Supplemental Figure 11), and deficient PCK1 expression was significantly associated with a larger tumor size and accelerated proliferation (Figure 7, A and D; Pearson correlation's coefficient, $r=-0.3935, P=0.0160$; Supplemental Table 2). In addition, downregulated PCK1 expression was significantly associated with poor tumor differentiation and prognosis (Supplemental Table 2). Moreover, the global $O$-GlcNAcylation was significantly higher in HCC tissues than in adjacent normal tissues (Figure 7, B and $\mathrm{E}$ and Supplemental Figure 11). Consistently, the p-AMPK and p-GFAT1 levels were reduced in HCC tissues (Figure 7B). We also observed a negative correlation between $\mathrm{PCK} 1$ protein-expression levels and $O-$-GlcNAcylation levels in HCC (Figure 7F, $r=-0.3565$, $P=0.0240$ ). In addition, the sWGA pull-down assay showed that enhanced CHK2 O-GlcNAcylation was associated with PCK1 downregulation (Figure 7, G and H and Supplemental Figure 11). Consistent with our in vitro data, we observed a strong negative correlation between p-Rb levels and PCK1 expression (Figure 7I, $r$ $=-0.3852, P=0.0168)$. In conclusion, this clinical validation supports the finding that PCK1 repression strengthens CHK2 O-GlcNAcylation and promotes tumor growth in human primary HCC.

\section{Discussion}

Emerging evidence has demonstrated that protein $\mathrm{O}$-GlcNAcylation plays a key role in tumorigenesis. Increased glucose flux through the HBP elevates UDP-GlcNAc, which enhances cellular $O$-GlcNAcylation. However, cancer cells are frequently faced with limited nutrients due to an insufficient and inappropriate vascular supply and rapid nutrient consumption (2). Previous metabolomics data demonstrated that the glucose concentrations in tumor tissues are generally lower than those in nontransformed tissues (31). The mechanisms underlying tumor growth during periods of metabolic stress through enhanced HBP activity and $O$-GlcNAcylation have not been fully elucidated. It remains unknown whether gluconeogenesis contributes to maintaining HBP-mediated $O$-GlcNAcylation in cancer cells under low nutrient conditions (5). Here, we present the first evidence that deficiency of the gluconeogenic enzyme PCK1 promotes cellular O-GlcNAcylation and tumorigenesis in HCC (Figure 7J). Moreover, we identify that CHK2 O-GlcNAcylation at T378 maintains its stability and oncogenic activity in hepatoma cells. Therefore, our study provides a link between PCK1 repression and hyper-O-GlcNAcylation underlying HCC oncogenesis.

Since the liver is the major site of gluconeogenesis during fasting, the role of gluconeogenesis in HCC has begun to draw more attention recently (32). During glucose starvation, cancer cells redistribute gluconeogenic intermediates to downstream pathways to facilitate their proliferation (5). PCK1, the rate-limiting enzyme of gluconeogenesis, is downregulated in $\operatorname{HCC}(24,33,34)$. The mammalian target of rapamycin complex 2 (mTORC2) and hepatitis B X-interacting protein (HBXIP) abrogate the expression of PCK1 by inhibiting the nuclear translocation of forkhead box protein O1 (FOXO1), a key transcriptional activator of the PCK1 gene $(35,36)$. In addition, posttranslational modifications such as sumoylation and acetylation of PCK1 are enhanced in HCC cells, which promotes its ubiquitination and subsequent degradation (37, 38). PCK1 mediates not only gluconeogenesis, but also glyceroneogenesis and TCA cataplerosis (39). In the present study, we found that PCK1 silencing promoted UDP-GlcNAc biosynthesis, thus enhancing cellular $O$-GlcNAcylation under a low-glucose condition ( $5 \mathrm{mM}$ glucose). Previous findings showed that the maximum rate of gluconeogenesis is approached at glucose concentrations under $5 \mathrm{mM}$, whereas high glucose levels inhibit gluconeogenesis $(40,41)$. Consistent with this observation, we did not detect any significant change in $O$-GlcNAcylation levels under high-glucose conditions (25 mM glucose) upon PCK1 depletion or overexpression, indicating that PCK1 regulated HBP flux and protein $O$-GlcNAcylation, depending on glucose availability. Moreover, the global O-GlcNAcylation levels of HCC tissues were obviously increased in Pck1-knockout mice, as compared with those in the WT mice after fasting. However, cell culture under $5 \mathrm{mM}$ glucose for 12 hours could not completely simulate the low-glucose conditions of tumor microenvironment. Given these limitations, further in vitro studies under different concentrations of glucose are needed to confirm that PCK1 modulates protein $O$-GlcNAcylation.

$O$-GlcNAcylation depends on OGT/OGA levels and the donor substrate UDP-GlcNAc. As a nutrient sensor, UDP-GlcNAc levels are dependent on glucose, amino acid, fatty acid, and nucleotide availability (42). Here, we revealed a dual role for PCK1 in regulating UDP-GlcNAc biosynthesis through OAA accumulation and the AMPK-GFAT1 axis under low-glucose conditions. On the one hand, the OAA levels decreased upon PCK1 overexpression in hepatoma cells, but accumulated in the liver tumors of LKO mice. LKO mice are unable to remove oxaloacetate from the TCA cycle (43). OAA is converted to Asp by GOT2, a key enzyme that plays a role in the TCA cycle and amino acid metabolism (44). Accordingly, amino acids synthesized from the TCA cycle (including Asp) were elevated in the blood of PCK1 KO mice (45). Pharmacologi- 


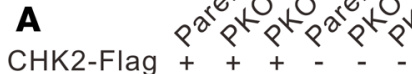

T378A-Flag - $-\quad+++$ $\mathrm{Ub}-\mathrm{HA}++++++\lg \mathrm{k} \mathrm{kDa}$

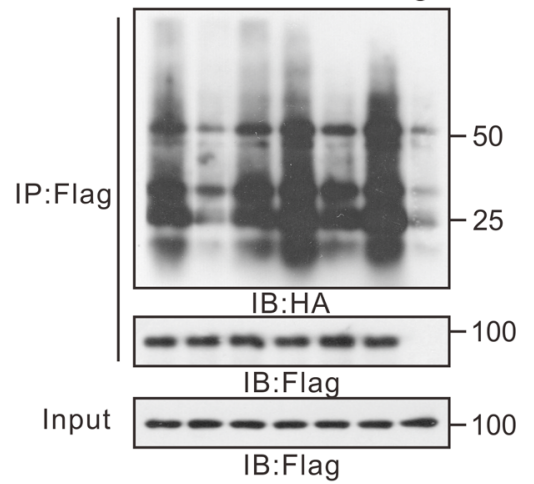

B

$\mathrm{CHX}$ (h) $\frac{\text { Parental }}{0122436} \frac{\text { PKO }}{0122436} \frac{\text { PKO+ST }}{0122436}$

CHK2-Flag $++++++++++++\mathrm{kDa}$

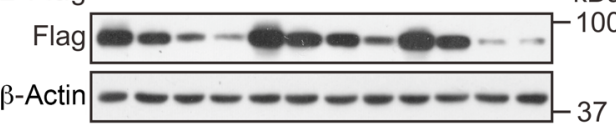

D

$\mathrm{CHX}$ (h) $\frac{\text { Parental }}{0122436} \frac{\mathrm{PKO}}{0122436} \frac{\mathrm{PKO}+\mathrm{ST}}{0122436}$ T378A-Flag $++++++++++++\mathrm{kDa}$

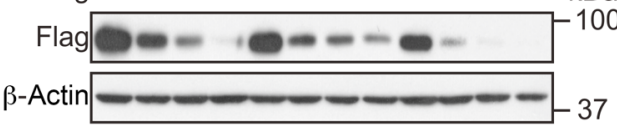

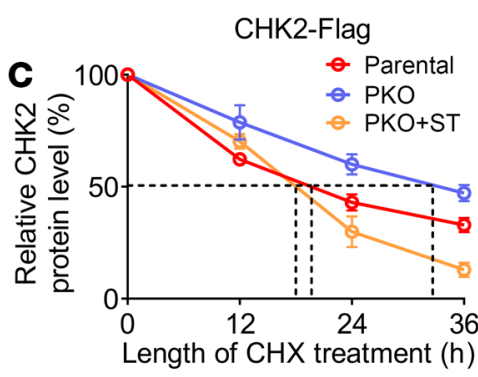

T378A-Flag

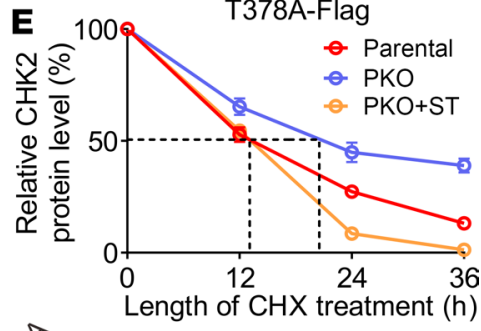

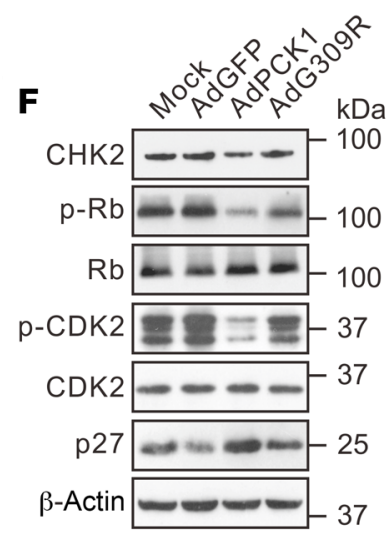

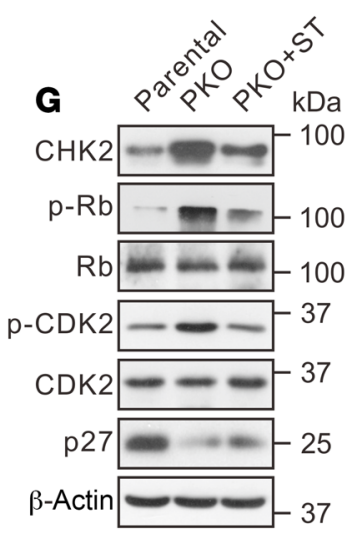

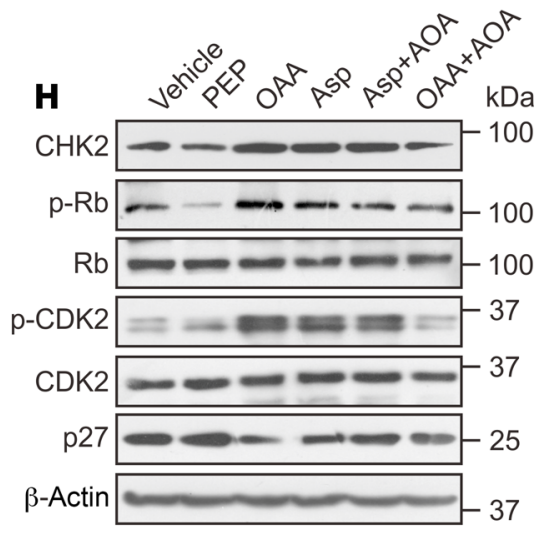
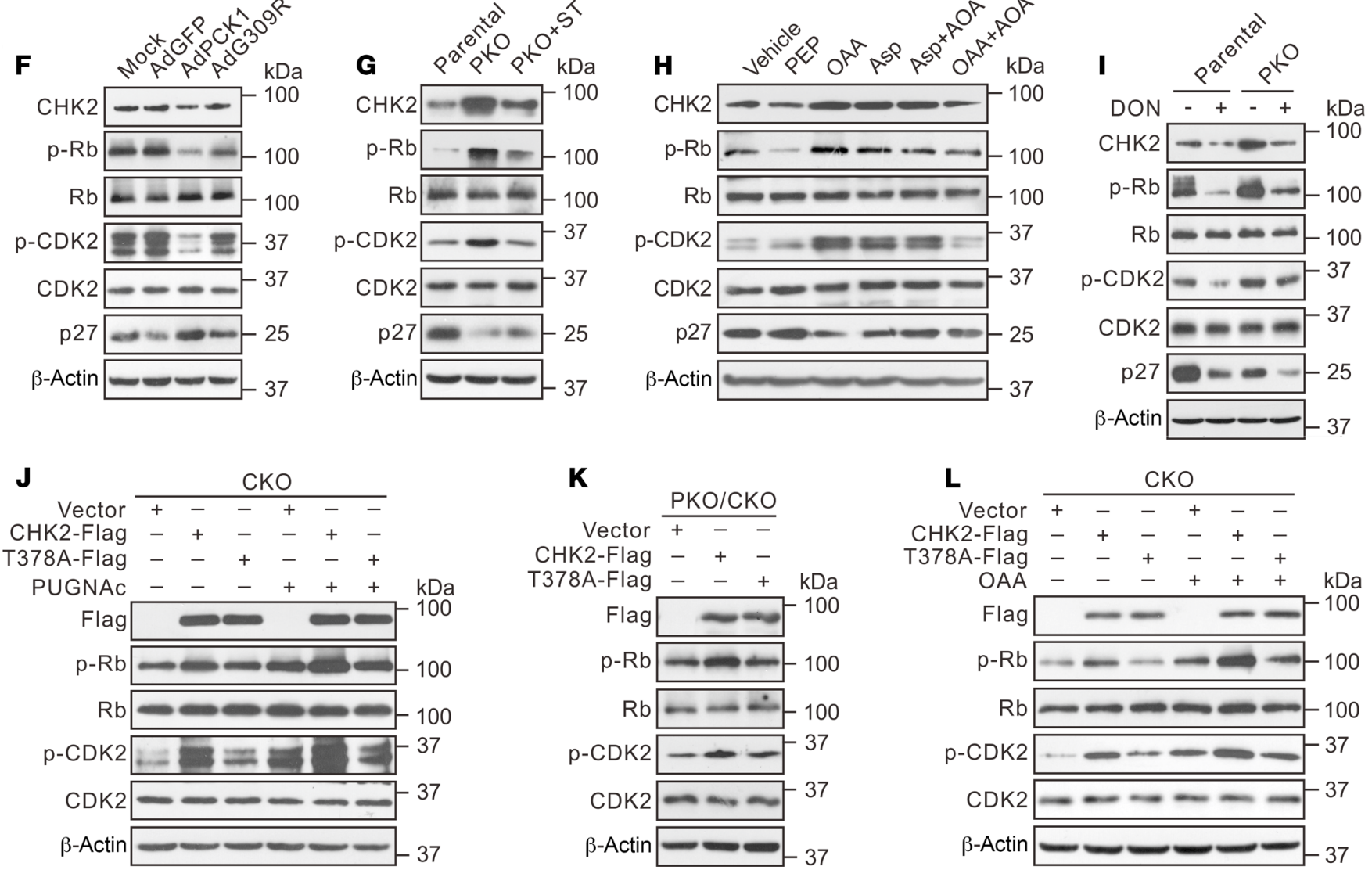

Figure 5. 0-CIcNAcylation at T378 stabilizes CHK2 and activates its downstream targets. (A) CHK2 ubiquitination in PKO cells in the presence of HA-tagged ubiquitin (Ub-HA). Half-life and quantitative analysis of Flag-tagged WT CHK2 (B, C) and T378A mutant CHK2 (D, E) in PKO cells. Cells were treated with $40 \mu \mathrm{M}$ cycloheximide ( $\mathrm{CHX}$ ) for the indicated time, and CHK2 levels was analyzed by immunoblotting. Data are representative of at least 3 independent experiments. Representative immunoblots of CHK2, p-Rb, p-CDK2, and p27 expression in PCK1-OE cells (F), PKO cells (G, I), or SK-Hep1 cells following the indicated treatments (H). (J, L) CKO cells (CHK2-knockout SK-Hep1 cells) were transfected with vectors CHK2-Flag or T378A-Flag, followed by treatment with $50 \mu \mathrm{M}$ PUCNAc for 24 hours (I) or $1 \mathrm{mM}$ OAA for 12 hours (L). Cells were lysed and analyzed by Western blotting. (K) PCK1/CHK2 double-knockout PLC/PRF/5 cells (PKO/CKO cells) were transfected with a CHK2-Flag or T378A-Flag expression vector, followed by immunoblotting.

cal or transcriptional inhibition of GOT2 suppressed hyper-O-GlcNAcylation induced by PCK1 deficiency in vitro and in vivo, which may represent an important therapeutic perspective for HCC treatment. These findings implied that the cataplerotic function of
PCK1 and the GOT2-mediated pathway are involved in regulating UDP-GlcNAc biosynthesis. Whether OAA-derived Asp is incorporated into UDP-GlcNAc via pyrimidine synthesis requires further study, based on stable isotope tracing. 
A

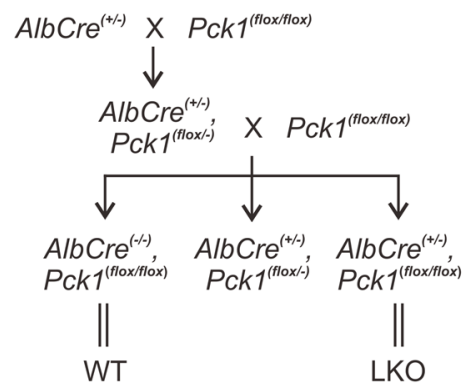

B

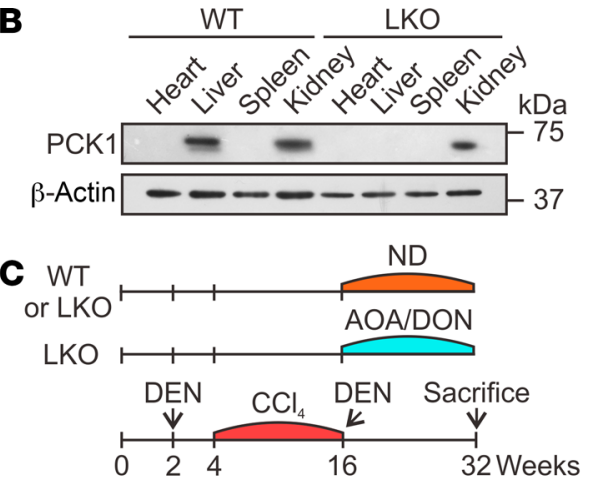

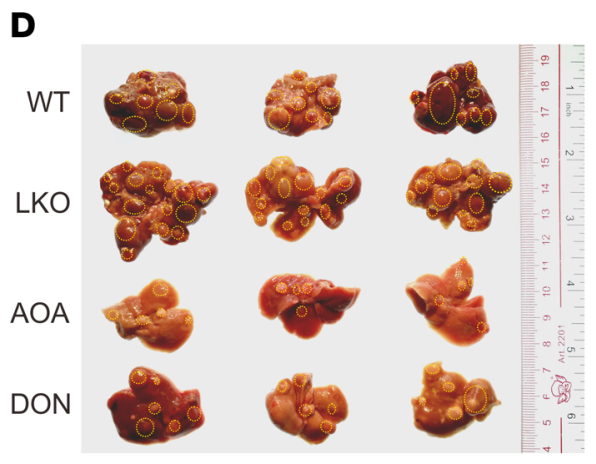

E WT

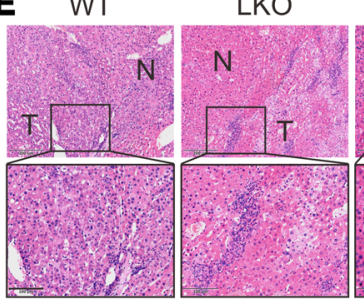

AOA

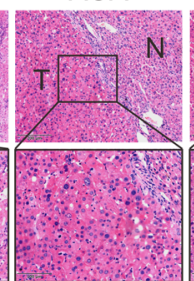

DON

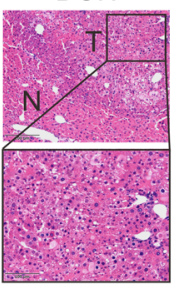

$\mathbf{F}$

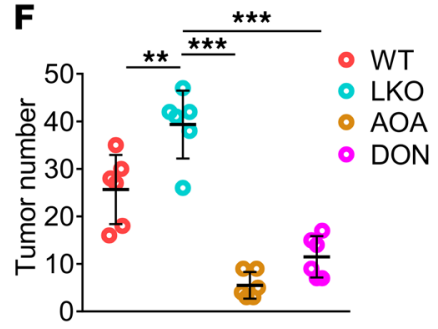

G

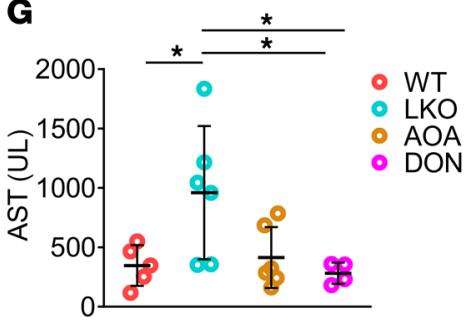

H

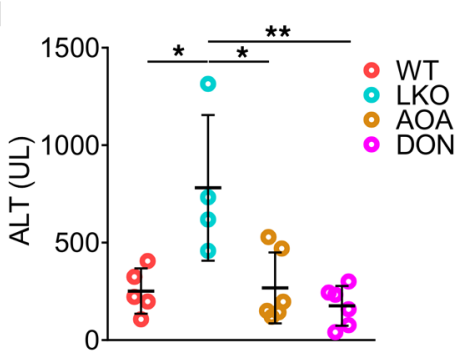

I

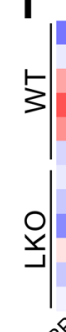

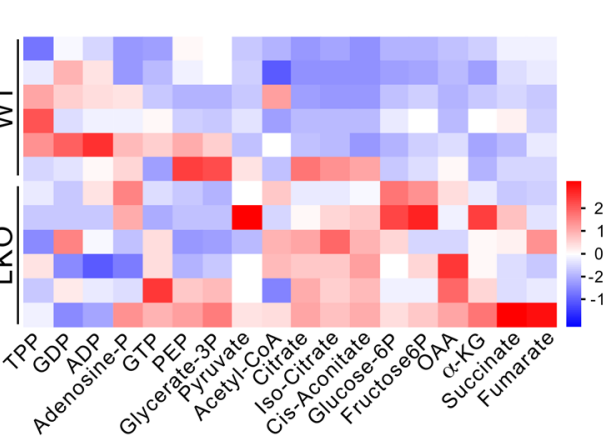

$\mathbf{K}$

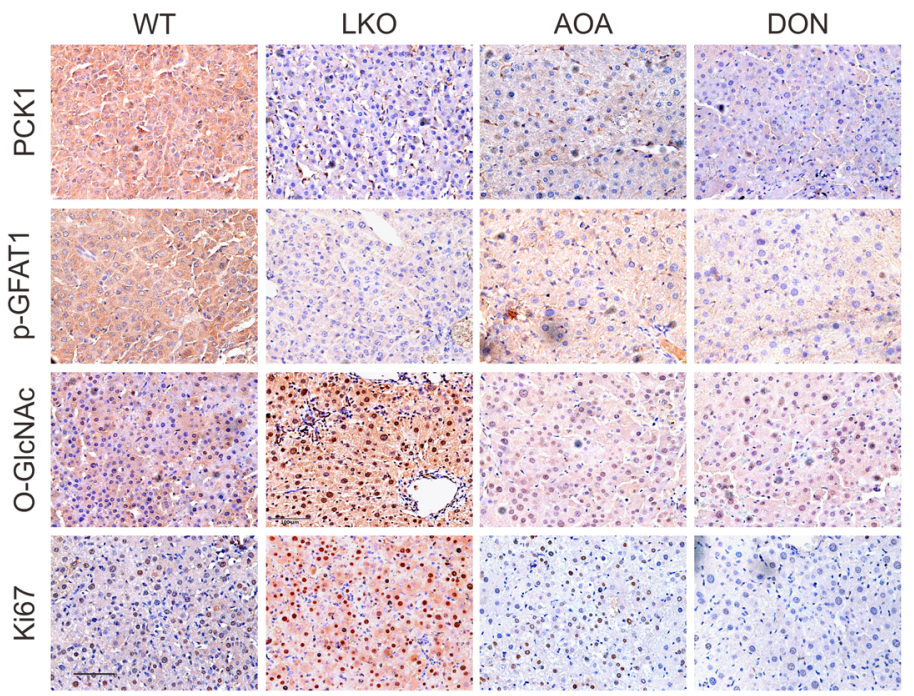

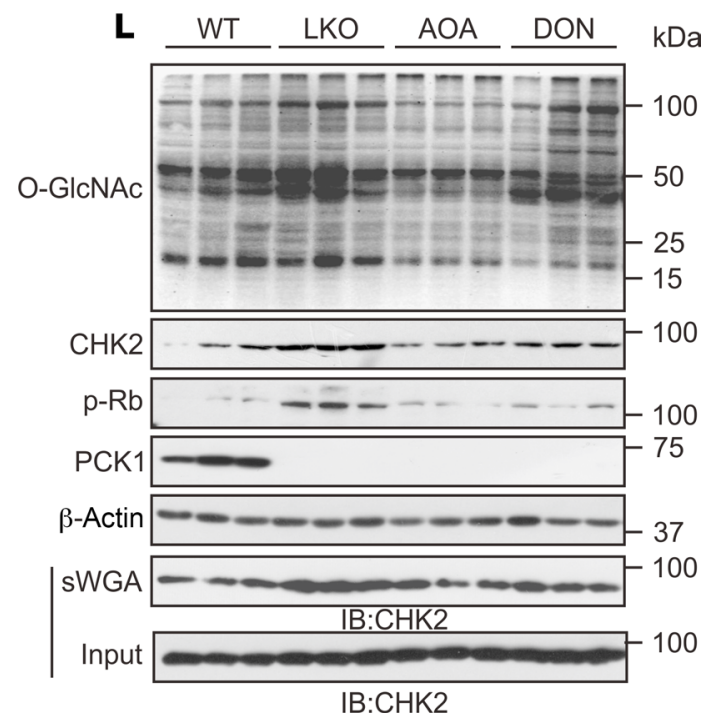

Figure 6. O-GIcNAcylation promotes DEN/CCI-induced hepatocellular carcinogenesis in PCK1-knockout mice. (A) Reproductive strategy for generating AlbCre ${ }^{(--)}$, $P c k 7^{(f / f)}(\mathrm{WT})$, and $A / b C r e^{(+/-)}, P c k 7^{(f / f)}$ (liver-specific knockout, LKO) mice. (B) PCK1 protein expression in WT and LKO mouse organs involving the heart, liver, spleen, and kidney were confirmed by immunoblotting. (C) Schematic representation of the experimental procedures used with WT and LKO mice. Mice were injected intraperitoneally with $75 \mathrm{mg} / \mathrm{kg} \mathrm{DEN}$ or $4 \% \mathrm{CCl}_{4}$ (every 3 days) as indicated, followed by combined administration of $5 \mathrm{mg} / \mathrm{kg} \mathrm{AOA} \mathrm{or} 1 \mathrm{mg} / \mathrm{kg} \mathrm{DON}$ (twice per week) for 16 weeks. Control mice were provided a normal diet (ND). Gross appearances (D) and hematoxylin and eosin staining (E) (scale bar: $100 \mu \mathrm{m})$ of liver samples with tumors, and the numbers of tumor nodules $(\mathbf{F}), n=6$ /group. Data are mean \pm SD. ${ }^{*} P<0.05,{ }^{* *} P<0.01,{ }^{* *} P<0.001,1$-way ANOVA followed by Tukey's test. The yellow dotted-line circles represent tumors. AST (C) and ALT (H) levels in mouse serum samples ( $n=6 /$ group). (I) Glycolysis-metabolite profiles, derived from liver tumors of WT or LKO mice, were determined by performing LC-MS/MS metabolomics assays. (J) Relative UDP-GlcNAc levels ( $n=6 /$ group). The indicated proteins in liver tumors were assessed by immunohistochemical labeling (K) (scale bar: $100 \mu \mathrm{m}$ ) and Western blotting (L). 
On the other hand, our previous work showed that enforced PCK1 expression leads to energy reduction and activates AMPK upon glucose deprivation in HCC (24). GFAT1 is an AMPK substrate, so we hypothesized that PCK1 may suppress HBP-mediated $O$-GlcNAcylation through the AMPK-GFAT1 axis. In support of this hypothesis, we found that PCK1 regulated GFAT1 phosphorylation and $O$-GlcNAcylation levels in an AMPK-dependent manner under glucose restrictions. As a rate-limiting enzyme of HBP, GFAT1 phosphorylation at Ser243 by AMPK diminishes its enzymatic activity (46), which decreases UDP-GlcNAc biosynthesis and $O$-GlcNAcylation levels. The AMPK-GFAT1 axis was also reported to regulate protein $O$-GlcNAcylation in angiogenesis and cardiac hypertrophy, indicating that this signal axis plays multiple physiological roles $(25,26)$. In addition, AMPK directly phosphorylated residue Thr444 of OGT, the enzyme responsible for O-GlcNAcylation (47). Therefore, PCK1 may regulate the HBP and $O$-GlcNAcylation through different mechanisms.

It is known that $O$-GlcNAcylation is crucial for cell-cycle regulation and DNA-damage responses (48). Several proteins that regulate the growth and proliferation of tumor cells can be $O$-GlcNAcylated. For example, the G1/S checkpoint protein Rb is heavily $O$-GlcNAcylated during the G1 phase (49). By characterizing the role of $O$-GlcNAcylation upon PCK1 deficiency, we uncovered CHK2 as novel target of OGT. CHK2, a cell-cycle checkpoint kinase, plays key roles in DNA-damage responses and cell-cycle progression. CHK2 can directly interact with $\mathrm{Rb}$ or activate the $\mathrm{p}$-CDK2/Cyclin A axis, thereby inducing the phosphorylation of $\mathrm{Rb}$ (50-52). The CHK2-modified $\mathrm{p}-\mathrm{Rb}$ promotes chromosomal instability and tumor progression in HCC (29). In addition, CHK2 activates $\mathrm{p}-\mathrm{CDK} 2 / \mathrm{Cyclin} \mathrm{A}$ and cell-cycle progression, resulting in hepatocyte growth (53). Rb is hyperphosphorylated in liver cancer $(54,55)$. Hyperphosphorylated Rb can no longer bind to E2F, thereby effectively allowing $\mathrm{E} 2 \mathrm{~F}$ transcription factors to activate transcription, and generating a cellular environment that is permissive for cell proliferation $(56,57)$. CHK2 is expressed in the nucleus in a subset of HCC and correlates with HCC progression (29). Several posttranslational modifications, including phosphorylation, ubiquitination, and acetylation, have been reported to be critical for CHK2 function (58-60). Herein, we identified Thr378 as a key $O$-GlcNAcylation site on CHK2 using LC-MS/MS. The loss of $O$-GlcNAcylation by the T378A mutation increased CHK2 ubiquitination, thus promoting its degradation. Moreover, we found that $O$-GlcNAcylation promoted CHK2 dimerization and activation, therefore enhancing $\mathrm{Rb}$ phosphorylation in PCK1-deficient hepatoma cells. Further structural analysis of $O$-GlcNAcylated CHK2 may help us understand how $O$-GlcNAcylation contributes to $\mathrm{CHK} 2$ activation.

Histopathology has revealed increased $O$-GlcNAcylation levels in HCC tumor tissues (61). Our findings not only provide an underlying mechanism whereby disrupted gluconeogenesis may activate the HBP and increase the availability of UDP-GlcNAc for $O$-GlcNAcylation under nutrient limitations, but also provide potential therapeutic targets for HCC. Preclinical evaluation of DON and AOA through the inhibition of glutamine metabolism has provided promising results for acute myeloid leukemia (21), high MYC-expressing atypical teratoid/rhabdoid tumors (62), and breast cancer (63). Here, we showed that both DON and AOA inhibited the growth of HCC in vitro and in vivo, largely by blocking HBP-mediated $O$-GlcNAcylation. These data suggest that DON and AOA inhibit cell growth through what we believe is a novel mechanism and provide a strong rationale for further clinical drug development, particularly for PCK1-deficient HCC.

In summary, we uncovered a link between gluconeogenesis disruption and $O$-GlcNAcylation upon glucose deprivation in HCC. We demonstrated that PCK1 deficiency can promote HBP-mediated UDP-GlcNAc biosynthesis through OAA accumulation and the AMPK-GFAT1 axis. Moreover, the OGT-mediated $O$-GlcNAcylation of CHK2 on Thr378 stabilizes CHK2 and promotes its oncogenic activity in HCC. The results of this study expand our understanding of PCK1 in hepatic carcinogenesis and indicate the potential of targeting HBP-mediated $O$-GlcNAcylation for HCC therapy.

\section{Methods}

Cell culture and treatment. PLC/PRF/5, SK-Hep1, Huh7, MHCC-97H, and HEK293 cells were cultured in DMEM (HyClone) supplemented with $10 \%$ FBS (Gibco), $100 \mathrm{U} / \mathrm{mL}$ penicillin, and $100 \mathrm{mg} / \mathrm{mL}$ streptomycin (HyClone) at $37^{\circ} \mathrm{C}$ in $5 \% \mathrm{CO}_{2}$. For low-glucose treatment, cells were briefly washed with PBS (DINGGUO, BF-0011) and then maintained in glucose-free medium (Gibco, 11966025) supplemented with $10 \%$ FBS and glucose at various concentrations for 12 hours. In addition, ST, TG, AOA, DON, or metformin was added to the medium, as indicated.

Animal studies. AlbCre ${ }^{(+/)}, P c k 1^{(\mathrm{fl} / \mathrm{fl})}$ (LKO) mice were generated from crosses between $\mathrm{AlbCre}{ }^{(+-)}$mice (Model Animal Research Center of Nanjing University) and $P c k 1^{(\mathrm{fl} / \mathrm{fl})}$ mice with a 129 background (Mutant Mouse Resource \& Research Centers, MMRRC:011950-UNC), as described previously (30), and AlbCre ${ }^{(-)}, P c k 1^{(\mathrm{fl} /}$ fl) (WT) mice were used as a control ( $n=6 /$ group). HCC was induced in mice by combined treatment with DEN $(75 \mathrm{mg} / \mathrm{kg})$ and $\mathrm{CCl}_{4}(2 \mathrm{~mL} /$ $\mathrm{kg}$, twice per week for 12 weeks), as described previously (64). At 16 weeks after $\mathrm{DEN} / \mathrm{CCl}_{4}$ treatment, the LKO mice were administered an intraperitoneal injection of $5 \mathrm{mg} / \mathrm{kg}$ AOA-hemihydrochloride (MilliporeSigma) or $1 \mathrm{mg} / \mathrm{kg}$ DON (MilliporeSigma) twice a week for 16 weeks. At 32 weeks, the mice were sacrificed after fasting for 12 hours, and liver tissues with tumors were collected for examination. Mouse serum ALT and AST were detected using an automatic biochemical analyzer (Catalyst One, IDEXX, USA).

For the orthotopic implantation model, BALB/c nude mice were randomly grouped ( $n=6$ /group). For each nude mouse, MHCC $97 \mathrm{H}$ cells $\left(1 \times 10^{5}\right.$, AdGFP-, AdPCK1-, AdG309R-, or mock-infected $)$ or $\mathrm{PLC} / \mathrm{PRF} / 5$ cells $\left(1 \times 10^{5}\right.$, parental, PCK1-knockout, or PCK1/CHK2double knockout) were suspended in a $50 \mu \mathrm{L}$ PBS/Matrigel (356234, BD Biosciences) mixture (1:1 ratio, vol/vol) and then implanted into the left liver lobe. At 4 weeks after implantation, all mice were sacrificed after fasting for 12 hours.

Clinical specimens. HCC samples and paired, adjacent normal liver tissues were obtained from the Second Affiliated Hospital of Chongqing Medical University between 2015 and 2018, with approval from the IRB of Chongqing Medical University.

Adenovirus production. The full-length cDNA fragment of PCK1 (NM_002591) or G309R (PCK1 mutation 925G $>$ A) was inserted into the pAdTrack-TO4 vector (gift from Tong-Chuan He, University of 
A

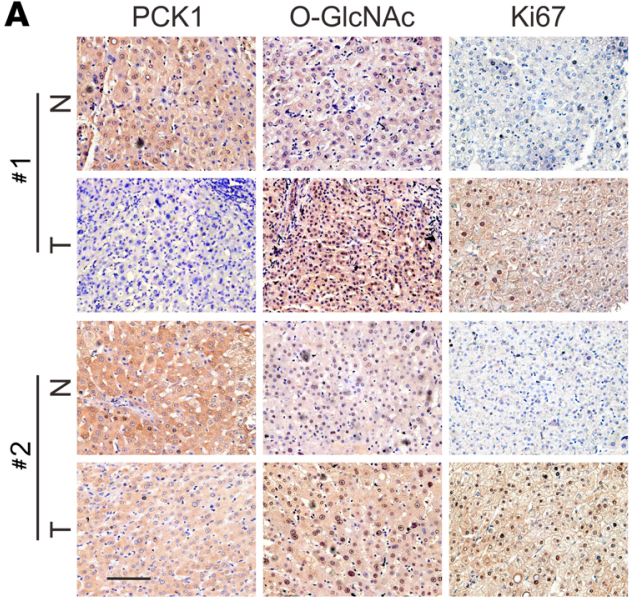

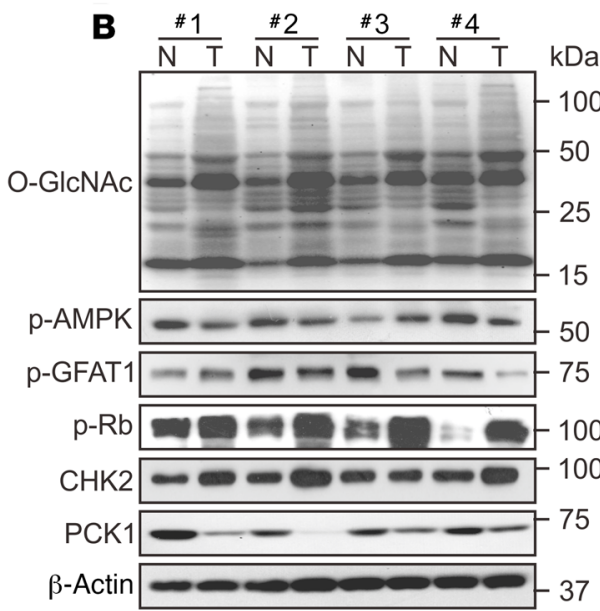

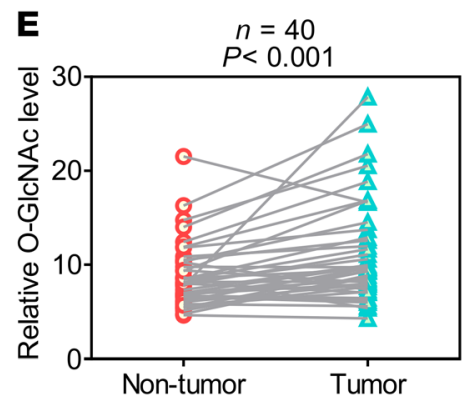

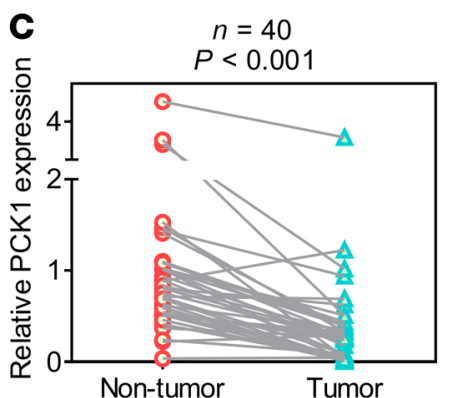

D

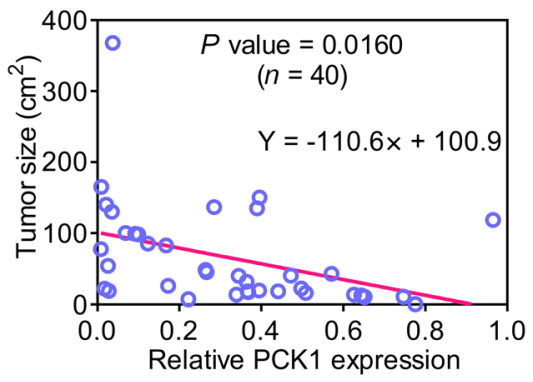

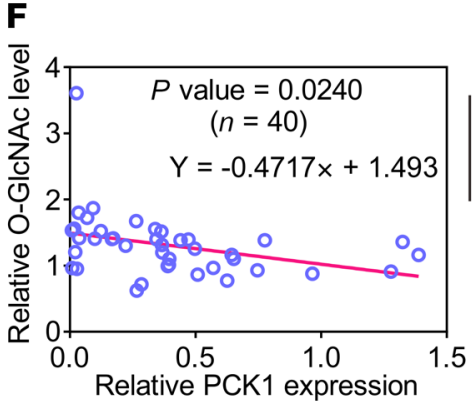

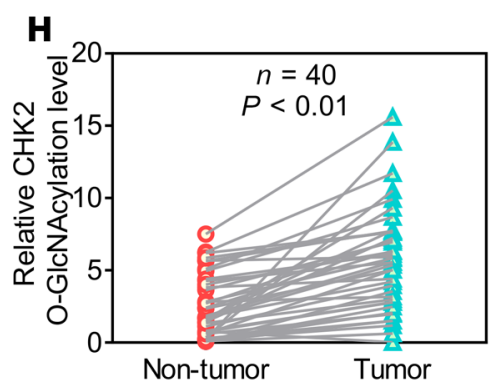

I
G
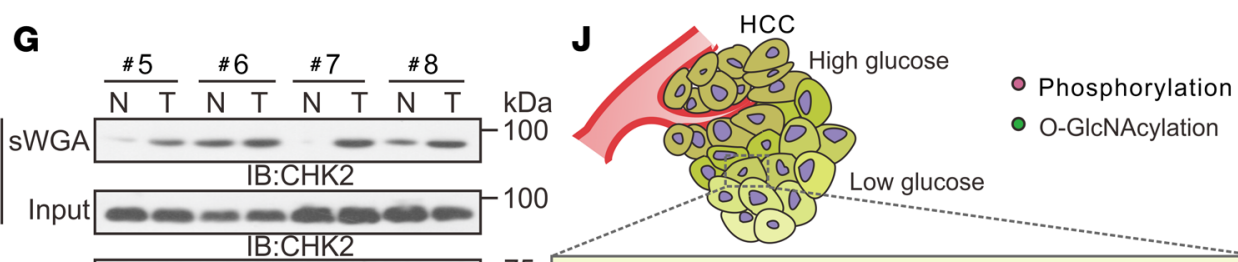
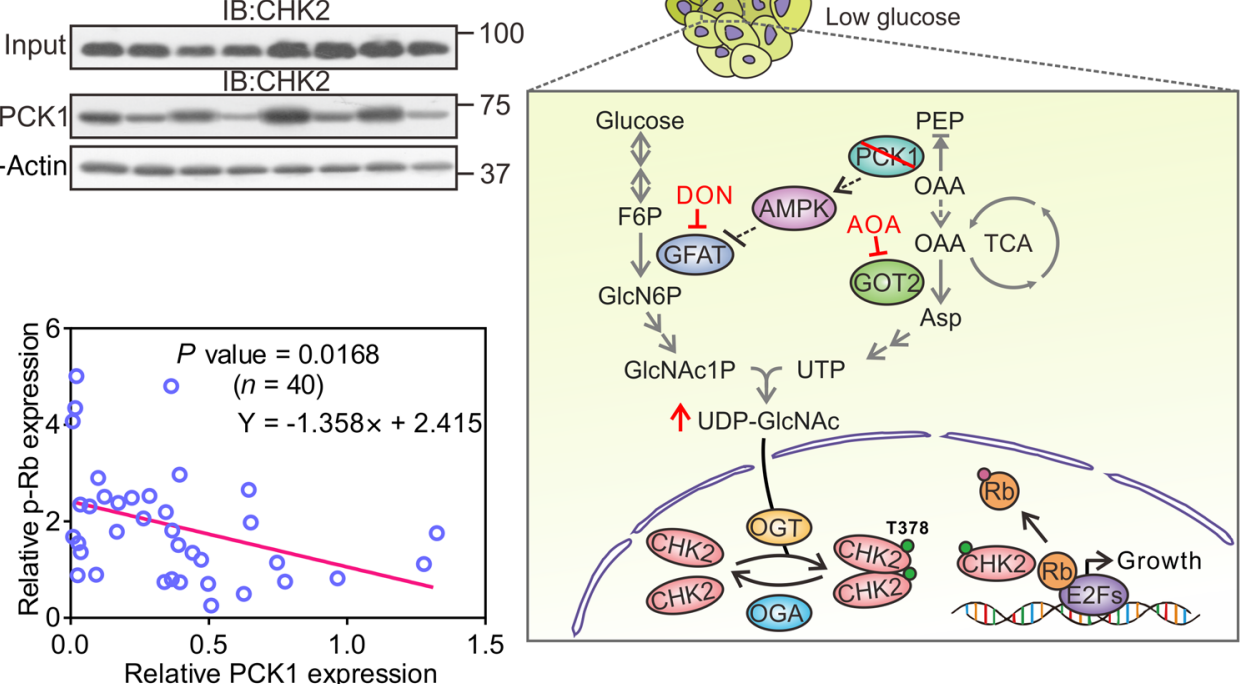

Figure 7. PCK1 deficiency strengthens protein $\mathbf{O}$-GIcNAcylation and correlates with human HCC growth. (A) IHC staining of PCK1, O-CICNAcylation, and Ki67 in clinical HCC samples. Scale bar: $100 \mu \mathrm{m}$. (B) Representative human HCC samples were indicated by immunoblots. (C) Relative PCK1 protein-expression levels were compared between nontumor tissues (NT) and tumors (T) from 40 patients with HCC (see also Supplemental Figure 11). Relative protein-expression levels were normalized to those in NT samples, followed by 2-tailed paired Student's $t$ test. (D) The correlation between HCC tumor sizes $(n=40)$ and PCK1 expression. Data are mean \pm SD. $P$ values were derived from Pearson's correlation coefficient $(r)$. (E) Relative 0 -GIcNAc levels of proteins in samples from 40 patients (see also Supplemental Figure 11). (F) Correlation analysis between PCK1 expression and 0 -GlcNAc levels $(n=40)$. Analysis of CHK2 O-GIcNAcylation in HCC tumors ( $n=40$, see also Supplemental Figure 11) by performing sWCA pull-down assays (C). CHK2 O-GIcNAcylation levels were quantified (H). (I) Correlation analysis between PCK1 and p-Rb expression in tumor tissues from 40 patients with HCC. (J) Molecular model for the role of PCK1 deficiency in regulating CHK2 O-GICNAcylation and HCC growth upon low glucose. In a low-glucose microenvironment, PCK1 ablation promotes oxaloacetate accumulation and GFAT1 activation to increase UDP-GIcNAc synthesis through the hexosamine-biosynthesis pathway. Increased $O$-CIcNAc modification enhances Thr378 O-GlcNAcylation in CHK2, which leads to its dimerization and Rb phosphorylation, and HCC cell proliferation. Inhibitors AOA and DON suppress HCC growth, indicating a unique potential for targeting $\mathrm{O}$-GICNAC signaling in the treatment of HCC. 
Chicago, Chicago, Illinois, USA). Recombinant adenovirals AdPCK1 and AdG309R were generated using the AdEasy system as described previously (24). The adenoviral AdGFP, expressing only GFP, was used as a control.

CRISPR/Cas9-mediated knockout cells. PCK1- or CHEK2-knockout cells (PKO or CKO cells) were established using the CRISPR-Cas9 system (gift from Ding Xue, the School of Life Sciences, Tsinghua University, Beijing, China), as previously described (24). Single-cell HCC clones stably expressing single guide RNA (sgRNA) sequences were propagated and validated by immunoblotting and DNA sequencing. The sequences of all oligonucleotides used to generate the knockout cell lines are listed in Supplemental Table 3.

Metabolite detection and analysis. Cells were washed twice with precooled physiological saline and metabolites were extracted with $400 \mu \mathrm{L}$ cold methanol/acetonitrile $(1: 1, \mathrm{vol} / \mathrm{vol})$ to remove the protein. The mixture was centrifuged for 20 minutes $\left(14,000 g, 4^{\circ} \mathrm{C}\right)$. The supernatant was dried in a vacuum centrifuge. For LC-MS analysis, the samples were redissolved in $100 \mu \mathrm{L}$ acetonitrile/water (1:1, vol/vol) solvent. Mouse liver tumor tissues $(60 \mathrm{mg}$ ) were extracted with $1 \mathrm{~mL}$ cold $90 \%$ methanol. The lysates were homogenized twice using an MP homogenizer $(24 \times 2,6.0 \mathrm{M} / \mathrm{S}, 60$ seconds). The homogenates were sonicated on ice. After centrifugation at $14,000 \mathrm{~g}$ for 20 minutes, the supernatant was dried and redissolved in $100 \mu \mathrm{L}$ acetonitrile/water (1:1, vol/vol) solvent.

Untargeted metabolomics profiling of PCK1-overexpressing cells was performed using ultra high-performance liquid chromatography (Agilent 1290 Infinity LC) coupled with quadrupole time-of-flight mass spectrometry (UHPLC-QTOF/MS) at Shanghai Applied Protein Technology Co., Ltd (65). UDP-GlcNAc levels were quantified by performing targeted LC-MS/MS analysis (Acquity UPLC I Class, Xevo G2-S QTof). TCA-derived metabolites were detected by UHPLC, using an Agilent 1290 Infinity LC column coupled to 5500 QTRAP system (AB SCIEX) at Shanghai Applied Protein Technology Co., Ltd.

${ }^{13} \mathrm{C}_{5}$-Glutamine labeling assay. PKO cells were incubated in DMEM (no glucose and no glutamine, Gibco, A1443001) supplemented with dialyzed fetal bovine serum containing $0.3 \mathrm{mg} / \mathrm{mL}$ [U-13C]glutamine (Cambridge Isotope Laboratories) and $1 \mathrm{mg} / \mathrm{mL}$ unlabeled glucose for 12 hours. PCK1-overexpressing Hep1-SK cells were preformed using the same method as above. The cells were washed with ice-cold saline, quenched with $80 \%$ methanol in $-80^{\circ} \mathrm{C}$ for 5 minutes, vortexed and centrifuged at $17,000 \mathrm{~g}$ for 15 minutes at $4^{\circ} \mathrm{C}$. The supernatant was collected and evaporated to dryness using a SpeedVac concentrator. Metabolites were vortexed and resuspended in $0.03 \%$ formic acid in analytical-grade water, and detected for use in liquid chromatography/triple quadrupole mass spectrometer (AB Sciex QTRAP 6500+) as previously described (66).

Immunoprecipitation. PKO or SK-Hep1 cells were transfected for 48 hours with a fusion vector expressing Flag-tagged fusion protein (OGT-Flag, CHK2-Flag, T378A-Flag, or T383A-Flag). Cells were treated with $50 \mu \mathrm{m}$ PUGNAc for 12 hours and resuspended in lysis buffer (50 mM Tris HCl, pH 7.4, $150 \mathrm{mM} \mathrm{NaCl}, 1 \mathrm{mM}$ EDTA, and 1\% Triton X-100) containing protease (Roche) and phosphatase (Beyotime Biotechnology) inhibitor cocktails. Precleared lysates were incubated overnight with an anti-FLAG M2 affinity gel (MilliporeSigma, A2220) at $4^{\circ} \mathrm{C}$.

For co-IP analysis, PKO or HEK293 cells were cotransfected with vectors expressing OGT-HA and either CHK2-Flag, $\Delta \mathrm{C}$-Flag, or $\Delta$ N-Flag. PKO cells were cotransfected with the CHK2-Flag/CHK2-
Myc, T378A-Flag/T378A-Myc, or CHK2-Flag/T378A-Myc vectors. Precleared cell lysates were incubated overnight with an anti-Flag or anti-HA antibody, and coupled with $40 \mu \mathrm{L}$ protein $\mathrm{A} / \mathrm{G}$ agarose beads. Immunoprecipitated complexes were eluted and subjected to immunoblotting using the indicated antibodies.

sWGA pull-down assay. Hepatic cells and liver tissues were lysed in a buffer containing $125 \mathrm{mM} \mathrm{NaCl}, 50 \mathrm{mM}$ Tris (pH 7.4), 5 mM EDTA, and $0.1 \% \mathrm{NP}-40$. The lysate was denatured in glycoprotein-denaturing buffer and digested with PNGase (NEB P0704S) to remove N-linked glycoproteins. Precleared lysates were incubated overnight with sWGA-conjugated agarose beads (Vector Laboratories). Precipitated complexes were eluted and immunoblotted with the indicated antibodies.

CHK2 O-GlcNAcylation site mapping. MS was performed to identify CHK2 O-GlcNAcylation sites, as previously described (67). Briefly, CHK2 was immunoprecipitated from PKO cells transfected with CHK2-Flag and subjected to 10\% SDS-PAGE for Coomassie blue staining. The band corresponding to CHK2 was excised from the gel and digested overnight with trypsin. Online LC-MS/MS system consisting of an Easy-nLC system and an Orbitrap Fusion Lumos Tribrid MS instrument (Thermo Fisher Scientific) equipped with a nanoelectrospray ion source were performed. The data from the raw MS files were analyzed against the UniProt database with MaxQuant software (version 1.5.2.8). The first-search peptide tolerance was $20 \mathrm{ppm}$ and the main-search peptide tolerance was $6 \mathrm{ppm}$. The MS/MS tolerance was $0.02 \mathrm{Da}$. The peptide-spectrum match and the false-discovery level was set to $1 \%$. Matches between runs were determined and the minimum score for modified peptides was set to 40 .

Quantitative analysis. Integral optical density (IOD) values were measured using Image-Pro Plus software (version 6.0, Media Cybernetics) to determine the intensity of protein expression. The IOD was calculated as the mean density times the area. The relative protein expression levels were normalized to an internal reference control, such as $\beta$-actin.

Statistics. All statistical analyses were performed using GraphPad Prism 7 (GraphPad Software Inc.). Data are represented as mean \pm SD. Unpaired or paired 2-tailed Student's $t$ test was used to compare 2 groups. One-way ANOVA followed by Tukey's test was used to compare more than 2 groups. Pearson's correlation coefficient was used to test linear correlations. Statistical significance was defined as $P$ less than 0.05. ${ }^{*} P<0.05,{ }^{* *} P<0.01,{ }^{* *} P<0.001$.

Study approval. All animal procedures were performed according to protocols approved by the IACUC at the Laboratory Animal Center of Chongqing Medical University. All procedures were also approved by the Research Ethics Committee of Chongqing Medical University (reference number 2017012). Clinical samples were collected from patients after obtaining informed consent in accordance with a protocol approved by the Second Affiliated Hospital of Chongqing Medical University.

For further details regarding the materials used, please refer to the online supplement (Supplemental Methods and Supplemental Tables 1-6).

\section{Author contributions}

NT, AH, and KW conceived and designed the study. J Xiang, CC, $\mathrm{RL}$, and DG performed most experiments and analyzed the data. LC and WZ performed CHK2 O-GlcNAcylation site mapping. HD and LT helped with data analysis. LL generated CHK2 mutants. QG, $\mathrm{XP}$, and J Xia assisted with mice experiments. LH performed molec- 
ular dynamic simulation. $\mathrm{KY}, \mathrm{BW}$, and $\mathrm{ZH}$ performed the isotopic tracing assay. J Xiang, KW, and NT wrote the manuscript with all authors providing feedback. The order of the co-first authors was assigned on the basis of their relative contributions to the study.

\section{Acknowledgments}

We would like to thank T-C He (University of Chicago) for providing the pAdEasy system and critical reading of the manuscript. We are grateful to Ding Xue (Tsinghua University) for supplying the CRISPR/Cas9 system. We also thank Bing Sun (Shanghai Institute of Biochemistry and Cell Biology) for providing the pLL3.7 vector. We thank Zhimin Lu (Zhejiang University) for suggestions and critical reading of the manuscript. This work was supported by the China National Natural Science Foundation (grants 82073251, 82072286, 81872270, U20A20392), the 111 Project (grant D20028), the Natural Science Foundation Project of Chongqing (grants cstc2018jcyjAX0254, cstc2019jscx-dxwtBX0019, cstc2019jcyj-msxmX0587), the Major National S\&T program (grant 2017ZX10202203-004), the Scientific Research Innovation Project for Postgraduate in Chongqing (grant CYS19191), the Kuanren Talents Program of The Second Affiliated Hospital of Chongqing Medical University, and the Science and Technology Research Program of Chongqing Municipal Education Commission (grants KJZD-M202000401, KJQN201900429).

Address correspondence to: Ni Tang, Kai Wang, or Ailong Huang, Key Laboratory of Molecular Biology for Infectious Diseases (Ministry of Education), Institute for Viral Hepatitis, Department of Infectious Diseases, The Second Affiliated Hospital, Chongqing Medical University, Chongqing, China. Phone: 86.23.68486780; Email: nitang@cqmu.edu.cn (NT); wangkai@cqmu.edu.cn (KW); ahuang@cqmu.edu.cn (AH).
1. Ward PS, Thompson CB. Metabolic reprogramming: a cancer hallmark even warburg did not anticipate. Cancer Cell. 2012;21(3):297-308.

2. Boroughs LK, DeBerardinis RJ. Metabolic pathways promoting cancer cell survival and growth. Nat Cell Biol. 2015;17(4):351-359.

3. Burén $S$ et al. Regulation of OGT by URI in response to glucose confers c-MYC-dependent survival mechanisms. Cancer Cell. 2016;30(2):290-307.

4. Lunt SY, Vander Heiden MG. Aerobic glycolysis: meeting the metabolic requirements of cell proliferation. Annu Rev Cell Dev Biol. 2011;27(1):441-464.

5. Grasmann G, et al. Gluconeogenesis in cancer cells - Repurposing of a starvation-induced metabolic pathway? Biochim Biophys Acta Rev Cancer. 2019;1872(1):24-36.

6. Wang Z, Dong C. Gluconeogenesis in cancer: function and regulation of PEPCK, FBPase, and G6Pase. Trends Cancer. 2019;5(1):30-45.

7. Nwosu ZC, et al. Identification of the consistently altered metabolic targets in human hepatocellular carcinoma. Cell Mol Gastroenterol Hepatol. 2017;4(2):303-323.

8. Montal ED et al. PEPCK coordinates the regulation of central carbon metabolism to promote cancer cell growth. Mol Cell. 2015;60(4):571-583.

9. Ma Z, Vosseller K. O-GlcNAc in cancer biology. Amino Acids. 2013;45(4):719-733.

10. Hanover JA, et al. O-GlcNAc in cancer: An oncometabolism-fueled vicious cycle. J Bioenerg Biomembr. 2018;50(3):155-173.

11. Hart GW, et al. Cycling of O-linked beta-N-acetylglucosamine on nucleocytoplasmic proteins. Nature. 2007;446(7139):1017-1022.

12. Zachara NE, Hart GW. O-GlcNAc a sensor of cellular state1-2: the role of nucleocytoplasmic glycosylation in modulating cellular function in response to nutrition and stress. Biochim Biophys Acta. 2004;1673(1-2):13-28.

13. Ferrer CM, et al. O-GlcNAcylation in cancer biology: linking metabolism and signaling. J Mol Biol. 2016;428(16):3282-3294.

14. Jóźwiak P, et al. O-GlcNAcylation and metabolic reprograming in cancer. Front Endocrinol (Lausanne). 2014;9;5:145.

15. Fardini Y, et al. O-GlcNAcylation: a new cancer hallmark? Front Endocrinol (Lausanne).
2013;12;4:99.

16. Nagel AK, Ball LE. Intracellular protein O-GlcNAc modification integrates nutrient status with transcriptional and metabolic regulation. Adv Cancer Res. 2015;126:137-166.

17. Love DC, Hanover JA. The hexosamine signaling pathway: deciphering the "O-GlcNAc Code". Sci STKE. 2005;2005(312):re13.

18. Chou T-Y, et al. c-Myc is glycosylated at threonine 58, a known phosphorylation site and a mutational hot spot in lymphomas. J Biol Chem 1995;270(32):18961-18965.

19. Park SY, et al. Snail1 is stabilized by O-GlcNAc modification in hyperglycaemic condition. EMBO J. 2010;29(22):3787-3796.

20. Sayat R, et al. O-GlcNAc-glycosylation of beta-catenin regulates its nuclear localization and transcriptional activity. Exp Cell Res. 2008;314(15):2774-2787.

21. Ricciardiello F, et al. Inhibition of the hexosamine biosynthetic pathway by targeting PGM3 causes breast cancer growth arrest and apoptosis. Cell Death Dis. 2018;9(3):377.

22. Sharma NS, et al. Targeting tumor-intrinsic hexosamine biosynthesis sensitizes pancreatic cancer to anti-PD1 therapy. JClin Invest. 2020;130(1):451-465.

23. Vieira P, et al. Novel homozygous PCK1 mutation causing cytosolic phosphoenolpyruvate carboxykinase deficiency presenting as childhood hypoglycemia, an abnormal pattern of urine metabolites and liver dysfunction. Mol Genet Metab. 2017;120(4):337-341.

24. Tuo L, et al. PCK1 negatively regulates cell cycle progression and hepatoma cell proliferation via the AMPK $/ \mathrm{p} 27^{\text {Kip } 1}$ axis. J Exp Clin Cancer Res. 2019;38(1):50.

25. Zibrova D, et al. GFAT1 phosphorylation by AMPK promotes VEGF-induced angiogenesis. Biochem J. 2017;474(6):983-1001.

26. Gélinas R, et al. AMPK activation counteracts cardiac hypertrophy by reducing O-GlcNAcylation. Nat Commun. 2018;9(1):374.

27. Pilié PG, et al. State-of-the-art strategies for targeting the DNA damage response in cancer. Nat Rev Clin Oncol. 2019;16(2):81-104.

28. Cai Z, et al. Structure and activation mechanism of the CHK2 DNA damage checkpoint kinase. Mol Cell. 2009;35(6):818-829.

29. Carloni V, et al. CHK2 overexpression and mislocalisation within mitotic structures enhances chromosomal instability and hepatocellular carcinoma progression. Gut. 2018;67(2):348-361.

30. She $\mathrm{P}$, et al. Phosphoenolpyruvate carboxykinase is necessary for the integration of hepatic energy metabolism. Mol Cell Biol. 2000;20(17):6508-6517.

31. Birsoy K, et al. Metabolic determinants of cancer cell sensitivity to glucose limitation and biguanides. Nature. 2014;508(7494):108-112.

32. Xu D, et al. The gluconeogenic enzyme PCK1 phosphorylates INSIG1/2 for lipogenesis. Nature. 2020;580(7804):530-535.

33. Ma R, et al. Switch of glycolysis to gluconeogenesis by dexamethasone for treatment of hepatocarcinoma. Nat Commun. 2013;4:2508.

34. Liu MX,et al. Metabolic reprogramming by PCK1 promotes TCA cataplerosis, oxidative stress and apoptosis in liver cancer cells and suppresses hepatocellular carcinoma. Oncogene. 2018;37(12):1637-1653.

35. Khan M, et al. mTORC2 controls cancer cell survival by modulating gluconeogenesis. Cell Death Discov. 2015;1:15016.

36. Shi $\mathrm{H}$, et al. The oncoprotein HBXIP suppresses gluconeogenesis through modulating PCK1 to enhance the growth of hepatoma cells. Cancer Lett. 2016;382(2):147-156.

37. Bian X, et al. Nur77 suppresses hepatocellular carcinoma via switching glucose metabolism toward gluconeogenesis through attenuating phosphoenolpyruvate carboxykinase sumoylation. Nature Commun. 2017;8:14420.

38. Jiang W, et al. Acetylation regulates gluconeogenesis by promoting PEPCK1 degradation via recruiting the UBR5 ubiquitin ligase. Mol Cell. 2011;43(1):33-44.

39. Beale E, et al. Disregulated glyceroneogenesis: PCK1 as a candidate diabetes and obesity gene. Trends Endocrinol Metab. 2004;15(3):129-135.

40. Rigoulet M, Leverve XM. Stimulation by glucose of gluconeogenesis in hepatocytes isolated from starved rats. Biochem J. 1987;245(3):661-668.

41. Ruan HB, et al. O-GlcNAc transferase/host cell factor $\mathrm{C} 1$ complex regulates gluconeogenesis 
by modulating PGC-1 $\alpha$ stability. Cell Metab. 2012;16(2):226-237.

42. Yang X, Qian K. Protein O-GlcNAcylation: emerging mechanisms and functions. Nat Rev Mol Cell Biol. 2017;18(7):452-465.

43. Beale EG, et al. PCK1 and PCK2 as candidate diabetes and obesity genes. Cell Biochem Biophys. 2007;48(2-3):89-95.

44. Altman BJ, et al. From Krebs to clinic: glutamine metabolism to cancer therapy. Nat Rev Cancer. 2016;16(10):619-634.

45. Hakimi P, et al. Phosphoenolpyruvate carboxykinase and the critical role of cataplerosis in the control of hepatic metabolism. Nutr Metab (Lond). 2005;2:33.

46. Eguchi S, et al. AMP-activated protein kinase phosphorylates glutamine: fructose-6-phosphate amidotransferase 1 at Ser243 to modulate its enzymatic activity. Genes Cells. 2009;14(2):179-189.

47. Bullen JW, et al. Cross-talk between 2 essential nutrient-sensitive enzymes: O-GlcNAc transferase (OGT) and AMP-activated protein kinase (AMPK). JBiol Chem. 2014;289(15):10592-10606.

48. Liu C, Li J. O-GlcNAc: a sweetheart of the cell cycle and DNA damage response. Front Endocrinol. 2018;9:415.

49. Wells L, et al. The E2F-1 associated retinoblastoma-susceptibility gene product is modified by O-GlcNAc. Amino Acids. 2011;40(3):877-883.

50. Inoue $\mathrm{Y}$, et al. Phosphorylation of $\mathrm{pRB}$ at
Ser612 by Chk1/2 leads to a complex between pRB and E2F-1 after DNA damage. EMBO J. 2007;26(8):2083-2093.

51. Pitts TM, et al. Targeting nuclear kinases in cancer: development of cell cycle kinase inhibitors. Pharmacol Ther. 2014;142(2):258-269.

52. Smith J, et al. The ATM-Chk2 and ATR-Chk1 pathways in DNA damage signaling and cancer. Adv Cancer Res. 2010;108:73-112.

53. Patra T, et al. Hepatitis $C$ virus mediated inhibition of miR-181c activates ATM signaling and promotes hepatocyte growth. Hepatology. 2020;71(3):780-793.

54. Chan HM, et al. Acetylation control of the retinoblastoma tumour-suppressor protein. Nat Cell Biol. 2001;3(7):667-674.

55. Burkhart DL, Sage J. Cellular mechanisms of tumour suppression by the retinoblastoma gene. Nat Rev Cancer. 2008;8(9):671-682.

56. Genovese C, et al. Cell cycle control and beyond: emerging roles for the retinoblastoma gene family. Oncogene. 2006;25(38):5201-5209.

57. van Harn T, et al. Loss of Rb proteins causes genomic instability in the absence of mitogenic signaling. Genes Dev. 2010;24(13):1377-1388.

58. Ahn JY, et al. Threonine 68 phosphorylation by ataxia telangiectasia mutated is required for efficient activation of Chk 2 in response to ionizing radiation. Cancer Res. 2000;60(21):5934-5936.

59. García-Limones C, et al. CHK2 stability is regu- lated by the E3 ubiquitin ligase SIAH2. Oncogene. 2016;35(33):4289-4301.

60. Zhang W, et al. SIRT1 modulates cell cycle progression by regulating CHK2 acetylation-phosphorylation. Cell Death Differ. 2020;27(2):482-496.

61. Satriano L, et al. Metabolic rearrangements in primary liver cancers: cause and consequences. Nat Rev Gastroenterol Hepatol. 2019;16(12):748-766.

62. Wang SZ, et al. Unbiased metabolic profiling predicts sensitivity of high MYC-expressing atypical teratoid/rhabdoid tumors to glutamine inhibition with 6-Diazo-5-Oxo-L-Norleucine. Clin Cancer Res. 2019;25(19):5925-5936.

63. Korangath P, et al. Targeting glutamine metabolism in breast cancer with aminooxyacetate. Clin Cancer Res. 2015;21(14):3263-3273.

64. Yang F, et al. GSTZ1-1 deficiency activates NRF2/IGF1R axis in HCC via accumulation of oncometabolite succinylacetone. $E M B O J$. 2019;38(15):e101964.

65. Lu L, et al. HJC0152 suppresses human nonsmall-cell lung cancer by inhibiting STAT3 and modulating metabolism. Cell Prolif. 2020;53(3):e12777.

66. Huang F, et al. Inosine monophosphate dehydrogenase dependence in a subset of small cell lung cancers. Cell Metab. 2018;28(3):369-382.

67. Peng C, et al. Regulation of the hippo-YAP pathway by glucose sensor O-GlcNAcylation. $\mathrm{Mol}$ Cell. 2017;68(3):591-604. 